ESAIM: PROCEEDINGS, October 2007, Vol.20, 170-194

Mohammed-Najib Benbourhim, Patrick Chenin, Abdelhak Hassouni \& Jean-Baptiste Hiriart-Urruty, Editors

\title{
GLIMPSES UPON QUASICONVEX ANALYSIS
}

\author{
JeAn-PAul PENOt ${ }^{1}$
}

\begin{abstract}
We review various sorts of generalized convexity and we raise some questions about them. We stress the importance of some special subclasses of quasiconvex functions. Dedicated to Marc Attéia
\end{abstract}

Résumé. Nous passons en revue quelques notions de convexité généralisée. Nous tentons de les relier et nous soulevons quelques questions. Nous soulignons l'importance de quelques classes particulières de fonctions quasiconvexes.

\section{INTRODUCTION}

Empires usually are well structured entities, with unified, strong rules (for instance, the length of axles of carts in the Chinese Empire and the Roman Empire, a crucial rule when building a road network). On the contrary, associated kingdoms may have diverging rules and uses. Because of their diversity, such outskirts are more difficult to describe than the central unified part and a global view may be out of reach. In this sense, the class of convex functions forms an empire, while the classes of generalized convex functions are outskirts.

In spite of the difficulty to find common features, it is the purpose of the present paper to review the main concepts of generalized convexity, to sketch some connections among these various generalizations and to raise some questions about them. Thus, it is just a slight complement to the recent monograph [115] which presents a much more complete view of the field of generalized convexity and generalized monotonicity.

When generalizing a concept, it is often the case that while some properties are lost, some new ones appear. As an example, let us mention that in passing from metric spaces to topological spaces one looses the use of sequences, but one gets the possibility of devising arbitrary products and initial or weak topologies. Another example, which is closer to our topic, is the case of starshaped subsets of a vector space $X$, a subset $S$ of $X$ being starshaped if for all $x \in S$ and $t \in[0,1]$ one has $t x \in S$. While an union of convex subsets is not convex in general, an union of starshaped subsets is always starshaped. Similarly, starshaped functions (i.e. functions which epigraphs are starshaped) are stable under infima. Thus, we may expect that, while most (but not all) of the "miraculous" properties of convex functions are lost in these various generalizations, some other properties may appear. For instance, we note that for any quasiconvex function $f$, and for any $c \in \mathbb{R}$, its truncation $f_{c}$ given by $f_{c}:=f \wedge c:=\min (f, c)$ is still quasiconvex (but in general it is no more convex when $f$ is convex). More generally, if $g$ is a quasiconvex function and $h: \mathbb{R} \rightarrow \mathbb{R}$ is a nondecreasing function, then $f:=h \circ g$ is still quasiconvex. The question of finding conditions ensuring that a quasiconvex function $f$ can be written in the form $h \circ g$ with $g$ convex and $h: \mathbb{R} \rightarrow \mathbb{R}$ nondecreasing is a long standing problem ( [97]). We raise a number of other questions, hoping that they will be stimuli for the field.

Several subjects are not dealt with in this short survey. As a sample of topics and references, let us mention: applications to mathematical economics ( $[186,217])$, applications to partial differential equations

\footnotetext{
1 Laboratoire de mathématiques appliquées CNRS UMR 5142, Faculté des sciences, BP 1155, 64013 Pau Cédex France
}

(C) EDP Sciences, SMAI 2007 
( [1,233, 235], [241], [317], [318]), asymptotic analysis ( [3], [4], [172], [213], [219]), calculus rules for subdifferentials ( [213], [238]), duality ([90], [180]- [184], [216], [218], [227], [229]- [230]), mechanics ( [102], [107], [206]), multicriteria optimization ( [115]), numerical issues ( [149], [226], [292], [293], [305], [307]), optimality conditions, regularization ( [239]), subdifferentials ( [189], [213]), variational convergences ( [23], [240], [256], [300])...Also, we do not venture in the wide world of abstract convex analysis although it is rich of promises and applications ( [195], [218], [227], [257]- [271], [286], [326]...). We hope that the bibliography we provide, albeit incomplete, will prove to be useful to the reader along with the ones in [115], [213] and [250] and that the tracks we delineate will be alluring and encouraging for further research.

\section{A SHORT REVIEW OF GENERALIZED CONVEXITY}

We devote this preliminary section to a review of some concepts of generalized convexity and their characterizations. For the proofs and credits we refer to [115] and its references. Several needs have led to weakened convexity assumptions, in particular in mathematical economics ( [109]); classifications are given in [82] and [83]. Among the classes of generalized convex functions, the most important one is the class of quasiconvex functions.

Definition 1. A function $f: X \rightarrow \overline{\mathbb{R}}:=\mathbb{R} \cup\{-\infty,+\infty\}$ on a vector space $X$ is said to be quasiconvex if for every $r \in \mathbb{R}$ its sublevel set $S_{f}(r):=\{f \leq r\}:=\{x \in X: f(x) \leq r\}$ is convex. Equivalently, $f$ is quasiconvex if for any $x_{0}, x_{1} \in X, t \in[0,1]$, one has

$$
f\left((1-t) x_{0}+t x_{1}\right) \leq f\left(x_{0}\right) \vee f\left(x_{1}\right):=\max \left(f\left(x_{0}\right), f\left(x_{1}\right)\right) .
$$

Condition (1) is clearly related to the convexity condition by the replacement of a convex combination of values by a supremum.

Example. Any nondecreasing (resp. nonincreasing) function $f: \mathbb{R} \rightarrow \overline{\mathbb{R}}$ is quasiconvex. More generally, a function $f: \mathbb{R} \rightarrow \overline{\mathbb{R}}$ is quasiconvex if, and only if, there is some $m \in \overline{\mathbb{R}}$ such that $f \mid(-\infty, m] \cap \mathbb{R}$ is nonincreasing and $f \mid[m,+\infty) \cap \mathbb{R}$ is nondecreasing. Such a property, sometimes called unimodality in connection with algorithms, is efficiently used in [89].

Example. Let $u: C \rightarrow \mathbb{R}$ be a function (interpreted as a utility function in mathematical economics) defined on a set $C$ (usually a cone $C$ of some n.v.s. $X$ ). Let $P$ be some convex cone or some vector subspace of the space $\mathbb{R}^{C}$ of functions from $C$ to $\mathbb{R}\left(P\right.$ is the set of prices, for instance $P=X^{*}$ when $C$ is a cone in some n.v.s. $X$ or $P$ is the dual cone of $C$ ). Let $v$ be the so-called inverse utility function given on $P$ by

$$
v(p):=\sup \{u(x): x \in C, p(x) \leq 1\} \quad p \in P .
$$

Then $v$ is quasi-convex on $P$ since for $p_{0}, p_{1} \in P$ and $t \in[0,1]$ and for $p_{t}:=(1-t) p_{0}+t p_{1}$ one has $\left\{p_{t} \leq 1\right\} \subset$ $\left\{p_{0} \leq 1\right\} \cup\left\{p_{1} \leq 1\right\}$, hence $v\left(p_{t}\right) \leq \max \left(v\left(p_{0}\right), v\left(p_{1}\right)\right)$.

The following stability properties are easy consequences of the definition. While the class of quasiconvex functions on $X$ is stable by suprema, this class is not preserved under sums. In fact, as observed by Crouzeix, a function $f: X \rightarrow \mathbb{R}_{\infty}:=\mathbb{R} \cup\{\infty\}$ on a normed vector space $X$ is convex if, and only if, for each $\ell \in X^{*}$ the function $x \longmapsto f(x)+\ell(x)$ is quasiconvex.

The continuity properties of quasiconvex functions are not as striking as the ones for convex functions; in particular one cannot expect a local Lipschitz property on the interiors of their domains. However, let us note the following mild continuity property which stems from the Baire property.

Lemma 2. Let $f: X \rightarrow \mathbb{R}_{\infty}:=\mathbb{R} \cup\{\infty\}$ be a l.s.c. quasiconvex function on a Banach space $X$. If $f$ is radially upper semicontinuous (u.s.c.) in the sense that its restriction to any line segment is u.s.c. at each point of its domain, then $f$ is continuous.

Quasiconvexity is an important tool for existence results. The reason is that a l.s.c. quasiconvex function is weakly l.s.c.; thus, if the space is reflexive and if moreover the function is coercive, it attains its infimum.

Some variants of quasiconvexity have some interest. 
Definition 3. A function $f: X \rightarrow \overline{\mathbb{R}}$ on a vector space $X$ is said to be strictly quasiconvex if for any $t \in] 0,1[$ and distinct $x_{0}, x_{1} \in X$, one has

$$
f\left((1-t) x_{0}+t x_{1}\right)<\max \left(f\left(x_{0}\right), f\left(x_{1}\right)\right) .
$$

It is said to be semistrictly quasiconvex if it is quasiconvex and if for any $t \in] 0,1\left[, x_{0}, x_{1} \in X\right.$, with $f\left(x_{0}\right) \neq$ $f\left(x_{1}\right)$, the preceding inequality holds.

Thus, a function is strictly quasiconvex if, and only if, it is quasiconvex and not constant on any proper line segment $[x, y]:=\{(1-t) x+t y: t \in[0,1]\}$. Clearly, a strictly quasiconvex function is quasiconvex and semistrictly quasiconvex. Also, any convex function is semistrictly quasiconvex (but not necessarily strictly quasiconvex, unless it is strictly convex); such a fact explains the change of terminology which occurred after the first contributions to the topic. More generally, if $f=h \circ g$, where $g: X \rightarrow \overline{\mathbb{R}}$ is convex or semistrictly quasiconvex and $h: \overline{\mathbb{R}} \rightarrow \overline{\mathbb{R}}$ is increasing, then $f$ is semistrictly quasiconvex. This fact provides a large amount of semistrictly quasiconvex functions.

Semistrictly quasiconvex functions retain some localization properties from the class of convex functions, such as the following one. We leave the easy proofs to the reader.

Proposition 4. (a) If $f: X \rightarrow \overline{\mathbb{R}}$ is a semistrictly quasiconvex function on a n.v.s. $X$, then any local minimizer of $f$ is a global minimizer.

(b) If $f: X \rightarrow \overline{\mathbb{R}}$ is an upper semicontinuous quasiconvex function whose local minimizers are global minimizers, then $f$ is semistrictly quasiconvex.

Characterizations of generalized convexity properties in terms of first order and second order derivatives have soon be obtained ( [21], [22], [24], [62], [61], [279]...) Let us give a characterization of quasiconvexity in terms of subdifferentials. Since we wish to dispose of as much flexibility as possible, we consider a notion which is quite loose. Here we call subdifferential the data of a multimap (or correspondence) $\partial: X \times \overline{\mathbb{R}}^{X} \rightrightarrows X^{*}$ for any normed vector space $X$, such that $\partial f(x)=\varnothing$ if $x \notin \operatorname{dom} f$ and such that

(M) $0 \in \partial f(x)$ if $x$ is a local minimizer of $f$,

(F) if $f$ is convex, then $x^{*} \in \partial f(x)$ iff $f(\cdot) \geq x^{*}(\cdot)-x^{*}(x)+f(x)$.

We will impose some other conditions later on. For instance, we may require $\partial$ is local in the sense that when $f$ and $g$ coincide on a neighborhood of $x$ one has $\partial f(x)=\partial g(x)$. One may also consider subdifferentials which are just defined for a class $\mathcal{X}$ of Banach spaces and for a subclass $\mathcal{F}(X)$ of $\overline{\mathbb{R}}^{X}$ or of the class $\mathcal{S}(X)$ of l.s.c. functions from $X$ into $\mathbb{R}_{\infty}:=\mathbb{R} \cup\{\infty\}$. Obviously, we need a limitation of the notion of subdifferential. We will impose that the subdifferential is not larger than a variant of the Clarke subdifferential (or the Clarke subdifferential itself if the reader prefers). This variant, which is adapted to questions in which rays and segments occur, is defined with the help of a generalized derivative called the dag derivative, given for $f: X \rightarrow \overline{\mathbb{R}}, x \in \operatorname{dom} f, v \in X$, by

$$
\begin{aligned}
f^{\dagger}(x, v) & :=\limsup _{t \rightarrow 0_{+}, y \rightarrow f x} \frac{1}{t}[f(y+t(v+x-y))-f(y)] \\
& :=\inf _{r>0} \sup _{(t, y) \in(0, r) \times B_{f}(x, r)} \frac{1}{t}[f(y+t(v+x-y))-f(y)] .
\end{aligned}
$$

where, as usual, $y \rightarrow_{f} x$ means that $y \rightarrow x$ with $f(y) \rightarrow f(x)$ and, for $r>0, B_{f}(x, r):=\{y \in B(x, r):$ $|f(y)-f(x)|<r\}$. Then the dag subdifferential is defined by setting

$$
\partial^{\dagger} f(x):=\left\{x^{*} \in X^{*}: x^{*} \leq f^{\dagger}(x, \cdot)\right\}
$$


Note that $f^{\dagger}$ majorizes both the radial (or Dini) upper derivative $f^{R}$ and the Clarke derivative $f^{C}$ given respectively (in the case $f$ is l.s.c.) by

$$
\begin{aligned}
f^{R}(x, v) & :=\limsup _{t \rightarrow 0_{+}} \frac{1}{t}[f(x+t v)-f(x)] \\
f^{C}(x, v) & :=\sup _{s>0} \limsup _{t \rightarrow 0_{+}, y \rightarrow f} \inf _{w \in B(v, s)} \frac{1}{t}[f(y+t w)-f(y)] .
\end{aligned}
$$

The inequality $f^{\dagger}(x, v) \geq f^{R}(x, v)$ is obvious. To prove the inequality $f^{\dagger}(x, v) \geq f^{C}(x, v)$ we use the following relation (obtained by taking $w:=v+x-y$ ) in which $0<r<s$

$$
\sup _{(t, y) \in(0, r) \times B_{f}(x, r)} \frac{1}{t}[f(y+t(v+x-y))-f(y)] \geq \sup _{(t, y) \in(0, r) \times B_{f}(x, r)} \inf _{w \in B(v, s)} \frac{1}{t}[f(y+t w)-f(y)],
$$

we take the infimum over $r \in(0, s)$ and then the supremum over $s>0$. When $f$ is locally Lipschitzian around $x, f^{\dagger}(x, \cdot)$ coincides with the Clarke derivative $f^{C}(x, \cdot)$, as easily seen.

A general, but not universal, means to define a subdifferential consists in setting, for $f: X \rightarrow \overline{\mathbb{R}}$ and $x \in X$ with $|f(x)|<+\infty$,

$$
\partial^{?} f(x):=\left\{x^{*} \in X^{*}: x^{*} \leq f_{x}^{?}\right\}
$$

where $f_{x}^{?}:=f^{?}(x, \cdot)$ is some approximation of $f$ at $x$, i.e. a positively homogeneous function $f_{x}^{?}: X \rightarrow \overline{\mathbb{R}}$ such that $f_{x}^{?} \geq f^{\prime}(x, \cdot)$, where $f^{\prime}(x, \cdot)$ is the lower (Hadamard or Dini-Hadamard or contingent or epi-) derivative of $f$ at $x$ given by

$$
f^{\prime}(x, u):=\liminf _{(v, t) \rightarrow\left(u, 0_{+}\right)} \frac{1}{t}(f(x+t v)-f(x)) \quad u \in X .
$$

However, the Fréchet subdifferential (see [35]), the Ioffe subdifferentials ( [134], [135]) and the limiting subdifferential ( [192], [193]) are not obtained in this way.

We will also frequently assume the following simplified mean value property which is an immediate consequence of the mean value theorem. In particular, it is satisfied if $X$ is an Asplund space and if $\partial$ is larger than the Fréchet subdifferential or if $X$ is a WCG Banach space and if $\partial$ is larger than the Hadamard subdifferential ( [35], [48], [169], [208]).

Definition 5. A subdifferential $\partial$ on a subclass $\mathcal{F}(X)$ of the class $\mathcal{S}(X)$ of l.s.c. functions on a given Banach space $X$ will be called quasi-valuable if for any $f \in \mathcal{F}(X)$ finite at $a \in X$ and any $b \in X$ with $f(b)>f(a)$ there exist $c \in[a, b):=[a, b] \backslash\{b\}$ and sequences $\left(c_{n}\right),\left(c_{n}^{*}\right)$ such that $\left(c_{n}\right) \rightarrow_{f} c, c_{n}^{*} \in \partial f\left(c_{n}\right)$ for each $n$ and

$$
\left\langle c_{n}^{*}, d-c_{n}\right\rangle>0 \quad \text { for all } d \in b+\mathbb{R}_{+}(b-a), n \in \mathbb{N} .
$$

It is valuable if for any $f \in \mathcal{F}(X)$ finite at $a \in X$ and any $b \in X \backslash\{a\}, r<f(b)$ there exist $c \in[a, b):=[a, b] \backslash\{b\}$ and sequences $\left(c_{n}\right),\left(c_{n}^{*}\right)$ such that $\left(c_{n}\right) \rightarrow_{f} c, c_{n}^{*} \in \partial f\left(c_{n}\right)$ for each $n$ and

$$
\begin{aligned}
\liminf _{n}\|d-c\|^{-1}\left\langle c_{n}^{*}, d-c_{n}\right\rangle & \geq\|b-a\|^{-1}(r-f(a)) \quad \text { for all } d \in b+\mathbb{R}_{+}(b-a), n \in \mathbb{N}, \\
\liminf _{n}\left\langle c_{n}^{*}, b-a\right\rangle & \geq r-f(a), \\
\lim _{n}\left\|c_{n}^{*}\right\| d\left(c_{n},[a, b]\right) & =0 .
\end{aligned}
$$

The properties which will serve to characterize the subdifferentials of generalized convex functions are the following ones. They can be defined for any multivalued operator (or multimap).

Definition 6. A multimap $F$ from a n.v.s. $X$ to its dual $X^{*}$ is said to be quasimonotone if for any $x, y \in X$

$$
\exists x^{*} \in F(x),\left\langle x^{*}, y-x\right\rangle>0 \Longrightarrow \forall y^{*} \in F(y) \quad\left\langle y^{*}, y-x\right\rangle \geq 0 .
$$


It is said to be pseudomonotone if for any $x, y \in X$

$$
\exists x^{*} \in F(x),\left\langle x^{*}, y-x\right\rangle \geq 0 \Longrightarrow \forall y^{*} \in F(y) \quad\left\langle y^{*}, y-x\right\rangle \geq 0 .
$$

It is said to be monotone if for any $x, y \in X$

$$
x^{*} \in F(x), y^{*} \in F(y) \Longrightarrow\left\langle x^{*}-y^{*}, x-y\right\rangle \geq 0 .
$$

Clearly,

$$
F \text { monotone } \Longrightarrow F \text { pseudomonotone } \Longrightarrow F \text { quasimonotone. }
$$

Moreover, $F$ is quasimonotone if, and only if, for any $x, y \in X$

$$
\forall x^{*} \in F(x), \forall y^{*} \in F(y) \quad\left\langle x^{*}, x-y\right\rangle \vee\left\langle y^{*}, y-x\right\rangle \geq 0 .
$$

Thus, as in the passage from convexity to quasiconvexity, in the passage from monotonicity to quasimonotonicity, the symbol + has been replaced with the symbol $\vee$ which stands for max. We also note that $F$ is pseudomonotone if and only if, for any $w, z \in X$

$$
\exists w^{*} \in F(w):\left\langle w^{*}, z-w\right\rangle>0 \quad \Longrightarrow \quad \forall z^{*} \in F(z):\left\langle z^{*}, z-w\right\rangle>0 .
$$

There is a close relationship between quasimonotonicity and monotonicity: an operator $M: X \rightrightarrows X^{*}$ is monotone iff for every $\ell \in X^{*}$ the multifunction $x \rightrightarrows M(x)+\ell$ is quasimonotone.

The characterization we have in view is as follows.

Theorem 7. ([11], [12], [211], [228]) Let $f: X \rightarrow \mathbb{R}_{\infty}$ be a l.s.c. function on a Banach space $X$ and let $\partial$ be a subdifferential on a class $\mathcal{F}(X)$ of functions containing $f$ such that $\partial f \subset \partial^{\dagger} f$. Among the following assertions one has the implications $(a) \Rightarrow(b) \Rightarrow(c)$. When $\partial$ is quasi-valuable on $\mathcal{F}(X)$, these three assertions are equivalent.

(a) $f$ is quasiconvex;

(b) $f$ is fully $\partial$-quasiconvex in the sense that $\operatorname{dom} f$ is convex and for any $x, y \in X, x^{*} \in \partial f(x)$ with $\left\langle x^{*}, y-x\right\rangle>0$, one has $f(y) \geq f(u)$ for any $u \in[x, y]$;

(c) $\partial f$ is quasimonotone.

Under a mild continuity assumption, condition (b) can be simplified. In the sequel we say that a function $f$ on $X$ is radially continuous if its restriction to any line segment of $X$ is continuous.

Corollary 8. Let $f: X \rightarrow \mathbb{R}_{\infty}$ be a radially continuous l.s.c. function on a Banach space $X$ and let $\partial$ be a quasi-valuable subdifferential on a subclass $\mathcal{F}(X)$ containing $f$, with $\partial f \subset \partial^{\dagger} f$. Then $f$ is quasiconvex if, and only if, it is $\partial$-quasiconvex in the sense that its domain is convex and $f$ satisfies the following condition:

(b') if $\left\langle x^{*}, y-x\right\rangle>0$ for some $x^{*} \in \partial f(x)$, then $f(y) \geq f(x)$.

The radial continuity requirement cannot be dropped.

Example. Let $f: \mathbb{R} \rightarrow \mathbb{R}$ be the l.s.c. function given by $f(x)=0$ if $x=-1$ or $x=1, f(x)=1$ for $x \in \mathbb{R} \backslash\{-1,1\}$. Then $f$ satisfies condition (b') for any subdifferential $\partial$ such that $\partial f \subset \partial^{\dagger} f$, but $f$ is not quasiconvex.

Let us note the following complement to the preceding results.

Lemma 9. If any local minimizer of $f: X \rightarrow \mathbb{R} \cup\{\infty\}$ with finite value is a global minimizer of $f$ and if $f$ is l.s.c. and d-quasiconvex for a quasi-valuable subdifferential $\partial$, then $f$ is quasiconvex.

One can deduce from Theorem 7 a subdifferential characterization of convexity.

Corollary 10. Let $\partial$ be a quasi-valuable subdifferential on a class $\mathcal{F}(X)$ of l.s.c. functions on $X$ stable by addition of continuous linear forms and such that $\partial f(x) \subset \partial^{\dagger} f(x)$ and $\partial(f+\ell)(x)=\partial f(x)+\ell$ for any $f \in \mathcal{F}(X), \ell \in X^{*}, x \in X$. Then $f$ is convex if, and only if, $\partial f$ is monotone. 
Since sublevel sets play a key role for quasiconvex functions, it is natural to look for a characterization in terms of normal cones to sublevel sets ( [18], [33]). In what follows we define the normal cone to a subset $S$ of $X$ at $x \in X$ as $N(S, x):=N^{\partial}(S, x):=\partial \iota_{S}(x)$, where $\iota_{S}$ is the indicator function of $S$ given by $\iota_{S}(x)=0$ if $x \in S,+\infty$ else. Then, for a function $f: X \rightarrow \overline{\mathbb{R}}$ finite at $x \in X$ we set

$$
N_{f}(x):=N\left(S_{f}(x), x\right)
$$

where $S_{f}(x):=S_{f}(f(x)):=\{f \leq f(x)\}$ is the sublevel set of $f$ for the level $f(x)$. We say that a subdifferential $\partial$ is local if $\partial f(x)=\partial g(x)$ whenever $f$ and $g$ coincide on a neighborhood of $x$ for any $x \in X$.

Theorem 11. ([16]) Let $\partial$ be a subdifferential on the class $\mathcal{S}(X)$ of l.s.c. functions on the Banach $X$. Then, for any $f \in \mathcal{S}(X)$, we have the implications $(a) \Rightarrow(b) \Rightarrow(c)$ among the following assertions. If $\partial$ is quasi-valuable and either local or contained in $\partial^{\dagger}$ and if $f$ is radially continuous, all these assertions are equivalent.

(a) $f$ is quasiconvex;

(b) if $\left\langle x^{*}, y-x\right\rangle>0$ for some $x, y \in X, x^{*} \in N_{f}(x)$, then $f(y)>f(x)$;

(c) $N_{f}(\cdot)$ is a quasimonotone multimap.

Proof. (a) $\Rightarrow$ (b) Let $x, y \in X, x^{*} \in N_{f}(x)$ be such that $\left\langle x^{*}, y-x\right\rangle>0$. Since $S_{f}(x)$ is convex, we cannot have $f(y) \leq f(x)$ as that means that $y \in S_{f}(x)$, hence, by condition $(\mathrm{F}),\left\langle x^{*}, y-x\right\rangle \leq 0$.

(b) $\Rightarrow$ (c) If $x, y \in X, x^{*} \in N_{f}(x), y^{*} \in N_{f}(y)$ are such that $\left\langle x^{*}, y-x\right\rangle>0$ and $\left\langle y^{*}, x-y\right\rangle>0$, assertion (b) cannot hold since it would imply $f(y)>f(x)$ and $f(x)>f(y)$.

$(\mathrm{c}) \Rightarrow\left(\right.$ a) Suppose $\partial$ is quasi-valuable and either local or contained in $\partial^{\dagger}$ and $f$ is radially continuous but not quasiconvex: for some $r \in \mathbb{R}$, the set $S:=S_{f}(r)$ is not convex. Then $\iota_{S}$ is l.s.c. but is not quasiconvex. By Theorem 7, $\partial \iota_{S}$ is not quasimonotone: there exist $x, y \in S$ and $x^{*} \in \partial \iota_{S}(x), y^{*} \in \partial \iota_{S}(y)$ such that $\left\langle x^{*}, y-x\right\rangle>0$ and $\left\langle y^{*}, x-y\right\rangle>0$. This is impossible if $f(x)=r=f(y)$ because then $S_{f}(x)=S=S_{f}(y)$ and $N_{f}(x)=N(S, x)=\partial \iota_{S}(x), N_{f}(y)=\partial \iota_{S}(y)$ while $N_{f}$ is quasimonotone. Suppose $f(x)<r$. Since $f$ is radially continuous, $x$ is an interior point to $S$, and $\iota_{S}$ is 0 on a neighborhood of $x$. Since $\partial$ is either local or contained in $\partial^{\dagger}$, one gets $x^{*}=0$, a contradiction with $\left\langle x^{*}, y-x\right\rangle>0$.

Now let us turn to the notions of pseudoconvexity and invexity. They are usually given under a differentiability assumption. In the sequel, given a subdifferential $\partial$ on a class $\mathcal{F}(X)$ of functions on $X$, we say that $x$ is a $\partial$ critical point of a function $f \in \mathcal{F}(X)$ if $0 \in \partial f(x)$.

Definition 12. A function $f: X \rightarrow \mathbb{R}_{\infty}$ is said to be pseudoconvex for a subdifferential $\partial$ (or, in short, d-pseudoconvex) if $\operatorname{dom} f$ is convex and if for any $x, y \in X$,

$$
\exists x^{*} \in \partial f(x),\left\langle x^{*}, y-x\right\rangle \geq 0 \quad \Longrightarrow \quad f(y) \geq f(x) .
$$

A function $f: X \rightarrow \mathbb{R}_{\infty}$ is said to be fully pseudoconvex for a subdifferential $\partial$ (or, in short, fully $\partial$-pseudoconvex) if $\operatorname{dom} f$ is convex and if for any $x, y \in X$

$$
\exists x^{*} \in \partial f(x),\left\langle x^{*}, y-x\right\rangle \geq 0 \quad \Longrightarrow \quad \forall u \in[x, y] \quad f(y) \geq f(u) .
$$

A function $f$ is said to be $\partial$-invex if any $\partial$-critical point $x$ of $f$ is a minimizer of $f$.

In particular, any local minimizer with finite value of a $\partial$-invex function is a (global) minimizer. It is easy to show that a function $f$ is invex if, and only if, there exists a map $v: \partial f \times X \rightarrow X$ such that for any $x \in \operatorname{dom} \partial f$, $x^{*} \in \partial f(x), y \in X$ one has $f(y)-f(x) \geq\left\langle x^{*}, v\left(x, x^{*}, y\right)\right\rangle$. Clearly, any convex function is $\partial$-pseudoconvex (but the converse is not true, as the next example shows) and any $\partial$-pseudoconvex function $f$ is $\partial$-invex; moreover, one easily sees that one can take $v$ such that $v\left(x, x^{*}, y\right)=\lambda\left(x, x^{*}, y\right)(y-x)$ for some $\lambda\left(x, x^{*}, y\right) \in \mathbb{R}_{+}$. The relationships of pseudoconvexity with quasiconvexity are not as simple.

Example: Let $f:=h \circ g$, where $g: X \rightarrow \mathbb{R}$ is convex and $h: \mathbb{R} \rightarrow \mathbb{R}$ is differentiable with a positive derivative. Then $f$ is $\partial$-pseudoconvex for the Fréchet and the Hadamard subdifferential. When $X=\mathbb{R}$, $f(x):=x^{3}+x=: h(x), g(x):=x, f$ is not convex. 
Example: The function $f: \mathbb{R} \rightarrow \mathbb{R}$ given by $f(x)=0$ for $x \leq 0, f(x)=x+1$ for $x>0$ is l.s.c. and $\partial$-pseudoconvex, for any subdifferential $\partial$ contained in $\partial^{\dagger}$ but $f$ is not of the type of the preceding example.

Example: The function $f: \mathbb{R} \rightarrow \mathbb{R}$ given by $f(0)=1, f(x)=0$ for $x \in \mathbb{R} \backslash\{0\}$ is $\partial$-pseudoconvex, for any subdifferential $\partial$ contained in $\partial^{\dagger}$, but it is not quasiconvex (note however that $f$ is not l.s.c.).

Example: The function $f: \mathbb{R} \rightarrow \mathbb{R}$ given by $f(x)=x^{3}$ is quasiconvex (since it is increasing), but it is not $\partial$-pseudoconvex (since it is not $\partial$-invex) for any subdifferential $\partial$ such that $f^{\prime}(x) \in \partial f(x)$.

Condition (8) is clearly a consequence of (9). Conversely, when $f$ is quasiconvex, condition (8) implies condition (9) since $f(y) \geq f(u)$ for any $u \in[x, y]$ when $f$ is quasiconvex and $f(y) \geq f(x)$. Thus, we can state:

Lemma 13. A quasiconvex function is fully $\partial$-pseudoconvex if, and only if, it is $\partial$-pseudoconvex.

For a quasi-valuable subdifferential a more complete relationship can be described.

Proposition 14. Let $\partial$ be a quasi-valuable subdifferential on a class $\mathcal{F}(X)$ of l.s.c. functions. Then, for every $f \in \mathcal{F}(X)$ such that $\partial f \subset \partial^{\dagger} f$, the following assertions are equivalent:

(a) $f$ is $\partial$-pseudoconvex;

(b) $f$ is fully $\partial$-pseudoconvex;

(c) $f$ is quasiconvex and $\partial-$ pseudoconvex.

Proof. (c) $\Rightarrow$ (b) has just been observed; (b) $\Rightarrow$ (a) is obvious.

(a) $\Rightarrow$ (c) If $x$ is a local minimizer of $f$ with finite value, we have $0 \in \partial f(x)$, hence $f(y) \geq f(x)$ for each $y \in X$. Since $\partial$-pseudoconvexity implies $\partial$-quasiconvexity, the result follows from Lemma 9 which shows that $f$ is quasiconvex.

Example. The l.s.c. function $f: \mathbb{R} \rightarrow \mathbb{R} \cup\{\infty\}$ given by $f(x)=0$, when $|x| \geq 1, f(x)=+\infty$ for $x \in(-1,1)$ shows that if one omits the requirement that the domain of $f$ is convex in the definition of $\partial$-pseudoconvexity, $f$ may not be quasiconvex.

For radially continuous functions an easy relationship between $\partial$-quasiconvexity and $\partial$-pseudoconvexity can be delineated.

Proposition 15. Let $\partial$ be a quasi-valuable subdifferential on a class $\mathcal{F}(X)$ of l.s.c. functions. Then, a radially continuous function $f \in \mathcal{F}(X)$ such that $\partial f \subset \partial^{\dagger} f$ is $\partial$-pseudoconvex if, and only if, it is $\partial$-quasiconvex and $\partial$-invex. In particular, a radially continuous function without critical points is $\partial$-pseudoconvex if, and only if, it is $\partial$-quasiconvex.

Proof. We have only to prove the sufficient condition. Let $f$ be radially continuous, $\partial$-quasiconvex and $\partial$-invex; then $\operatorname{dom} f$ is convex. Let $x \in \operatorname{dom} \partial f, y \in X$ and $x^{*} \in \partial f(x)$ be such that $\left\langle x^{*}, y-x\right\rangle \geq 0$. If $x^{*}=0$, $x$ is a critical point of $f$, hence a minimizer of $f$ and in particular $f(x) \leq f(y)$. If $x^{*} \neq 0$ we can find a unit vector $u$ such that $\left\langle x^{*}, u\right\rangle>0$. For $t>0$ let $y_{t}:=y+t u$. Then $\left\langle x^{*}, y_{t}-x\right\rangle>0$, so that, by $\partial$-quasiconvexity, we have $f\left(y_{t}\right) \geq f(x)$. Since $f$ is radially continuous, we get $f(y) \geq f(x)$. Thus, $f$ is $\partial$-pseudoconvex.

Now let us deal with the relationships between pseudoconvexity of a function and pseudomonotonicity of its subdifferential.

Theorem 16. Let $f: X \rightarrow \overline{\mathbb{R}}$ be l.s.c. and let $\partial$ be a quasi-valuable subdifferential such that $\partial f \subset \partial^{\dagger} f$. Then assertion (a) which follows implies assertion (b). If $f$ is radially continuous, (a) and (b) are equivalent:

(a) $f$ is $\partial$-pseudoconvex

(b) $\partial f$ is pseudomonotone.

One can give examples showing that one cannot drop the radial continuity assumption in the implication (b) $\Rightarrow$ (a) which precedes.

There are important variants of the preceding concepts, either involving strict inequalities or cyclic features; we refer to [25], [74], [115] for studies of such concepts. 


\section{Approximate convexity}

Approximate convexity is another kind of generalized convexity in which some fuzziness appears. It has been introduced and studied in [197] and characterized in [199]; we refer to these papers for the proofs of statements in the present section. Some variants are given in [49], [168], [198], [201], [202], [220].

Definition 17. A function $f: X \rightarrow \mathbb{R} \cup\{+\infty\}$ is said to be approximately convex around $\bar{x} \in X$ if for any $\varepsilon>0$ there exists $\delta>0$ such that for any $x, x^{\prime} \in B(\bar{x}, \delta)$ and any $t \in[0,1]$ one has

$$
f\left(t x+(1-t) x^{\prime}\right) \leq t f(x)+(1-t) f\left(x^{\prime}\right)+\varepsilon t(1-t)\left\|x-x^{\prime}\right\| .
$$

Clearly, convex functions and functions which are strictly differentiable at $\bar{x}$ are approximately convex around $\bar{x} \in X$. It can be shown that approximately convex functions retain some of the nice properties of convex functions [197]. In particular, they are continuous on segments contained in their domains and have radial derivatives. They are locally Lipschitzian in the interiors of their domains. Approximately convex functions on an open subset of an Asplund space are generically Fréchet differentiable ( [203]).

Proposition 18. ([197]) The set of approximately convex functions around $\bar{x} \in X$ is stable under addition, multiplication by positive numbers and finite suprema.

Characterizations of approximate convexity have been obtained in [17], [49], [72], [199]. They use concepts introduced by Spingarn [289] (under the name of strict submonotonicity) and studied [198], [201], [202], [209], [220], [255].

Definition 19. A multimapping $M: X \rightrightarrows X^{*}$ is approximately monotone around $\bar{x} \in \operatorname{dom}(M)$ provided that for each $\varepsilon>0$ there exists $\rho>0$ such that

$$
\forall x_{i} \in B(\bar{x}, \rho), x_{i}^{*} \in M\left(x_{i}\right), i=1,2 \quad\left\langle x_{1}^{*}-x_{2}^{*}, x_{1}-x_{2}\right\rangle \geq-\varepsilon\left\|x_{1}-x_{2}\right\|
$$

Theorem 20. ([199]) Given a subdifferential $\partial$ and $f$ l.s.c., let $\bar{x} \in \operatorname{dom} f$. Suppose $\partial f \subset \partial^{\dagger} f$. Then, among the following assertions, one has the implications $(a) \Rightarrow(b) \Rightarrow(c) \Leftrightarrow\left(c^{\prime}\right) \Rightarrow(d)$.

If moreover $\partial$ is valuable on $X$, all these assertions are equivalent.

(a) $f$ is approximately convex around $\bar{x}$;

(b) $\forall \varepsilon>0 \exists \rho>0$ such that for all $x \in B(\bar{x}, \rho), v \in B(0, \rho)$ one has

$$
f^{\dagger}(x, v) \leq f(x+v)-f(x)+\varepsilon\|v\|
$$

(c) $\forall \varepsilon>0, \exists \rho>0$ such that for all $x \in B(\bar{x}, \rho), x^{*} \in \partial f(x),(u, t) \in S_{X} \times(0, \rho)$ one has

$$
\left\langle x^{*}, u\right\rangle \leq \frac{f(x+t u)-f(x)}{t}+\varepsilon
$$

(c') $\forall \varepsilon>0 \exists \rho>0$ such that $\forall x \in B(\bar{x}, \rho), \forall x^{*} \in \partial f(x), \forall v \in B(0, \rho)$ one has

$$
\left\langle x^{*}, v\right\rangle \leq f(x+v)-f(x)+\varepsilon\|v\| ;
$$

(d) $\partial f$ is approximately monotone around $\bar{x}$.

Corollary 21. The preceding assertions (a), (b), (c), (d) are equivalent when

(i) $X$ is an arbitrary Banach space and $\partial$ is the Clarke or the Ioffe subdifferential;

(ii) $X$ is an Asplund space and $\partial$ is the Fréchet subdifferential or the Hadamard subdifferential.

Moreover, they are equivalent to the variant of assertion (b) obtained by replacing $f^{\dagger}$ by $f^{C}$ (and, if $X$ is an Asplund space, by $f^{\prime}$, the lower derivative of $f$ ). 
One may wonder whether there are some passages from approximate convexity to the classical forms of generalized convexity considered in the preceding section. One realizes that one cannot expect too much since any function of class $C^{1}$ is approximately convex but not necessarily quasiconvex or pseudoconvex. For the reverse direction, one notes that the function $f: \mathbb{R} \rightarrow \mathbb{R}$ given by $f(x)=-x$ for $x \leq 0, f(x)=-2 x$ for $x>0$ is pseudoconvex and quasiconvex, but not approximately convex around 0 . On the other hand, the coincidence of most classical subdifferentials of nonsmooth analysis on the class of approximately convex functions is an advantage. In particular, for approximately convex functions, a mean value theorem with the Fréchet and the Hadamard subdifferentials is available in any Banach space. Thus, one can drop for such functions the assumption that the subdifferential is quasi-valuable in the characterizations of the preceding section.

\section{QUASI-AFFINE AND PSEUDO-AFFINE FUNCTIONS}

A map $F: X \rightarrow Y$ (or multimap) between two vector spaces is called convexiphore if, for every convex subset $C$ of $X$, the set $F(C)$ is convex in $Y$. Equivalently, $F$ is convexiphore if, and only if, it transforms segments into convex subsets. For $Y=\mathbb{R}$, the following characterization is immediate, using the fact that a subset of $\mathbb{R}$ is convex if, and only if, it is an interval.

Lemma 22. A function $f: X \rightarrow \mathbb{R}$ is convexiphore if, and only if, it is quasi-affine, i.e. both quasiconvex and quasiconcave.

Quasi-affine functions are also called quasimonotonic ( [190]) or, more frequently, quasilinear (but this last choice does not take into account the fact that functions which are both convex and concave are affine functions, not linear functions). For $X=\mathbb{R}$, we easily see that $f$ is quasiaffine if, and only if, it is either nondecreasing or nonincreasing. For the rest of this section we suppose $X$ is finite dimensional. We make use of the following result.

Lemma 23. ([182]) A lower semicontinuous function $f: X \rightarrow \mathbb{R}$ is quasi-affine if, and only if, there exist a continuous linear form $g$ on $X$ and a lower semicontinuous nondecreasing function $h: \mathbb{R} \rightarrow \mathbb{R}$ such that $f=h \circ g$.

We deduce from that result a characterization of continuous quasi-affine functions.

Proposition 24. A continuous function $f: X \rightarrow \mathbb{R}$ is quasiaffine if, and only if, there exist a continuous linear form $g$ on $X$ and a continuous nondecreasing function $h: \mathbb{R} \rightarrow \mathbb{R}$ such that $f=h \circ g$.

Proof. The condition is clearly sufficient. Let $f: X \rightarrow \mathbb{R}$ be continuous and quasi-affine. By the preceding lemma, we can find a continuous linear form $g$ on $X$ and a lower semicontinuous nondecreasing function $h$ : $\mathbb{R} \rightarrow \mathbb{R}$ such that $f=h \circ g$. When $g=0, f$ is constant and then we can take for $h$ a constant function. When $g \neq 0, g$ is open and surjective. Then $h$ is continuous whenever $f$ is continuous: for any open subset $G$ of $\mathbb{R}$ the set $h^{-1}(G)=g\left(f^{-1}(G)\right)$ is open.

Now, given a quasi-valuable subdifferential $\partial$, we turn to $\partial$-pseudo-affine functions, i.e. functions $f$ which are both $\partial$-pseudoconvex and $\partial$-pseudoconcave (i.e. such that $-f$ is $\partial$-pseudoconvex). The differentiable case is considered in [32, Cor. 1.2], [37], [38], [154]. These references provide interesting, non trivial examples of pseudo-affine functions; in particular fractional functions are noticeable pseudo-affine functions and quadratic pseudo-affine functions can be characterized. See also [154] and [166] for the nonsmooth case. Here we use an arbitrary subdifferential $\partial$ and we suppose that when $f=h \circ g$ for some non null continuous linear form $g$ on $X$ and some continuous function $h: \mathbb{R} \rightarrow \mathbb{R}$, the following conditions are satisfied:

(C1) $\partial(h \circ g)(x) \subset \partial h(g(x)) \circ g$;

$(\mathrm{C} 2)$ if $0 \in \partial h(g(x))$ for some $x \in X$, then $0 \in \partial(h \circ g)(x)$.

This last property is obviously satisfied when $\partial h(g(x)) \circ g \subset \partial(h \circ g)(x)$. In particular, it is satisfied for the Fréchet and the Hadamard subdifferentials. Property (C1) is also satisfied for these subdifferentials(see [223]). 
Proposition 25. Let $f: X \rightarrow \mathbb{R}$ be a continuous, nonconstant function and let $\partial$ be a quasi-valuable subdifferential such that $\partial f \subset \partial^{\dagger} f$ and $\partial(-f) \subset \partial^{\dagger}(-f)$. If condition (C1) is satisfied, then assertion (a) below implies assertion (b); if condition (C2) is satisfied, the reverse implication holds:

(a) $f$ is $\partial$-pseudoaffine;

(b) there exist a continuous linear form $g$ on $X$ and a continuous $\partial$-pseudo-affine, nondecreasing function $h: \mathbb{R} \rightarrow \mathbb{R}$ such that $f=h \circ g$.

Proof. (a) $\Rightarrow$ (b) Let $f: X \rightarrow \mathbb{R}$ be continuous, nonconstant and $\partial$-pseudoaffine. Since $\partial$ is quasi-valuable and $\partial f \subset \partial^{\dagger} f, \partial(-f) \subset \partial^{\dagger}(-f)$, by Proposition 14, $f$ is quasiconvex and quasiconcave. By Proposition 24, there exist a continuous linear form $g$ on $X$ and a continuous nondecreasing function $h: \mathbb{R} \rightarrow \mathbb{R}$ such that $f=h \circ g$. Since $f$ is nonconstant, we have $g \neq 0$. Let us show that $h$ and $-h$ are $\partial$-pseudoconvex. Since $h$ and $-h$ are continuous, nondecreasing and nonincreasing respectively, hence quasiconvex, it suffices to show they are $\partial$-invex. Let $r, s \in \mathbb{R}$ be such that $0 \in \partial h(r), 0 \in \partial(-h)(s)$. Then, by $(\mathrm{C} 2)$, for any $w, x \in X$ such that $g(w)=r, g(x)=s$, we have $0 \in \partial f(w)$ and $0 \in \partial(-f)(x)$. Since $f$ is $\partial$-pseudo-affine, for every $u \in X$ one has $f(u) \geq f(w)$ and $-f(u) \geq-f(x)$. Since $g$ is surjective, it follows that for every $t \in \mathbb{R}$ one has $h(t) \geq h(r)$, $-h(t) \geq-h(s)$. Thus $h$ and $-h$ are invex, hence $\partial$-pseudoconvex.

(b) $\Rightarrow$ (a) Suppose $f=h \circ g$ with $g \in X^{*}$ and $h: \mathbb{R} \rightarrow \mathbb{R}$ a continuous $\partial$-pseudo-affine, nondecreasing function. If $g=0, f$ is constant, a trivial case we exclude. When $g \neq 0$, condition (C1) ensures that if $x, y \in X, x^{*} \in \partial f(x)$ are such that $\left\langle x^{*}, y-x\right\rangle \geq 0$, we can find $r^{*} \in \partial h(g(x))$ such that $x^{*}=r^{*} \circ g$. Then, $\left\langle r^{*}, g(y)-g(x)\right\rangle=\left\langle x^{*}, y-x\right\rangle \geq 0$ and since $h$ is $\partial$-pseudoconvex, we get $f(y)-f(x)=h(g(y))-h(g(x)) \geq 0$. Thus $f$ is $\partial$-pseudoconvex. Similarly, we obtain that $-f$ is $\partial$-pseudoconvex.

Question. What can be said when $f$ is $\partial$-pseudo-affine and just lower semicontinuous?

\section{SUbDifFERENTIALS AND CONJUGACIES}

In this section, we draw the attention on the nice properties of subdifferentials associated with conjugacies. In particular, a reversibility property of the type

$$
y \in \partial^{c} f(x) \Longleftrightarrow x \in \partial^{c} f^{c}(y)
$$

is enjoyed by such subdifferentials, thus extending the main feature of the Legendre transform to the case of a conjugacy $f \mapsto f^{c}$. On the other hand, such subdifferentials may not satisfy the conditions we imposed in section 2 .

Given a coupling function $c: X \times Y \rightarrow \overline{\mathbb{R}}$ between two sets $X, Y$, one defines the conjugacy $f \mapsto f^{c}$ from $\overline{\mathbb{R}}^{X}$ to $\overline{\mathbb{R}}^{Y}$ by

$$
f^{c}(y):=-\inf _{x \in X}(f(x)-c(x, y)) \quad f \in \overline{\mathbb{R}}^{X}, y \in Y .
$$

The reverse conjugacy is given by

$$
g^{c}(x):=-\inf _{y \in Y}(g(y)-c(x, y)) \quad g \in \overline{\mathbb{R}}^{Y}, x \in X .
$$

Note that the writing we adopt takes into account the classical conventions $(-\infty)+(+\infty)=+\infty, r-s:=r+(-s)$ for $r, s \in \overline{\mathbb{R}}$. One may have $-\inf _{x \in X}(f(x)-c(x, y)) \neq \sup _{x \in X}(c(x, y)-f(x))$. The subdifferential of $f \in \overline{\mathbb{R}}^{X}$ at $x \in X$ associated with $c$ is defined by

$$
y \in \partial^{c} f(x) \Longleftrightarrow f(x)=-\left(f^{c}(y)-c(x, y)\right) .
$$

When $f(x)$ is finite, the relation $f(x)=-\left(f^{c}(y)-c(x, y)\right)$ ensures that $f^{c}(y)$ and $c(x, y)$ are finite and then

$$
y \in \partial^{c} f(x) \Longleftrightarrow f(x)+f^{c}(y)=c(x, y) .
$$


If moreover $f(x)=f^{c c}(x):=\left(f^{c}\right)^{c}(x)$, one gets

$$
y \in \partial^{c} f(x) \Longleftrightarrow x \in \partial^{c} f^{c}(y) .
$$

The special cases of the radiant and co-radiant dualities deserve some attention in view of their simplicity. Let us say that a function $f$ on a vector space $X$ is radiant if its sublevel sets are either empty or are convex subsets containing 0. Equivalently, a function is radiant if it is quasiconvex and if it attains its minimum at 0. For example, $f$ is radiant when $f$ can be written $f=h \circ g$, where $g: X \rightarrow \mathbb{R}$ is a nonnegative convex function null at 0 and $h: \mathbb{R} \rightarrow \mathbb{R}$ is nondecreasing. When $X$ and $Y$ are locally convex topological vector spaces in duality, it is natural to study l.s.c. radiant functions. They are characterized by the property $f=f^{c_{\wedge} c_{\wedge}}$, where $c_{\wedge}$ is the coupling function defined by

$$
c_{\wedge}(x, y):=-\iota_{\{y \geq 1\}}(x) \quad(x, y) \in X \times Y,
$$

where $\iota_{S}$ is the indicator function of a subset $S$ of $X$. In such a case, for $f \in \overline{\mathbb{R}}^{X}$, one has

$$
f^{c_{\wedge}}(y)=-\inf _{x \in\{y \geq 1\}} f(x)=\sup _{x \in\{y \geq 1\}}-f(x),
$$

so that $f^{c_{\wedge}}$ is radiant. For $f$ finite at $x$, one has

$$
\begin{array}{rlr}
y \in \partial^{c_{\wedge}} f(x) & \Longleftrightarrow(y(x) \geq 1, \forall w \in\{y \geq 1\} \quad f(w) \geq f(x)) \\
& \Longleftrightarrow(y(x) \geq 1, \forall v \in\{f<f(x)\} \quad y(v)<1)
\end{array}
$$

Thus, if $x$ is not a local minimizer of $f$, one has $y(x)=1$ for every $y \in \partial^{c \wedge} f(x)$, hence

$$
y \in \partial^{c_{\wedge}} f(x)(y(x)=1, \forall v \in\{f<f(x)\} \quad y(v-x)<0) \Longleftrightarrow\left(y(x)=1, y \in \partial^{*} f(x)\right),
$$

where $\partial^{*}$ is the Greenberg-Pierskalla subdifferential of $f$ at $x$ (which is defined by $y \in \partial^{*} f(x) \Longleftrightarrow y(v-x)<0$ for all $v \in\{f<f(x)\}$ ). The radiant duality is derived from a polarity (see [217], [316] for instance). In fact, setting for a subset $A$ of $X$,

for all $r \in \mathbb{R}$ one has

$$
A^{\wedge}:=\{y \in Y: \forall x \in A y(x)<1\},
$$

$$
\left\{f^{c_{\wedge}} \leq r\right\}=\{f<-r\}^{\wedge}
$$

since $y \in\left\{f^{c_{\wedge}} \leq r\right\}$ iff for all $x \in\{y \geq 1\}$ one has $-f(x) \leq r$ iff $y(x)<1$ for all $x \in\{f<-r\}$, iff $y \in\{f<-r\}^{\wedge}$.

Several variants exist, but the associated subdifferentials are more loosely connected with known subdifferentials as the ones in [213], [236].

Two other subdifferentials are adapted to quasiconvex functions (and have some connections with duality theory, but not as tight as the preceding case). They are the lower subdifferential, or Plastria subdifferential given by

$$
\begin{aligned}
y \in \partial^{<} f(x) & \Longleftrightarrow(\forall w \in\{f<f(x)\} \quad f(w) \geq f(x)+\langle y, w-x\rangle) \\
& \Longleftrightarrow(\forall w \in X \quad f(w) \geq f(x)-\langle y, x-w\rangle \vee 0)
\end{aligned}
$$

and the infradifferential, or Gutiérrez subdifferential, given by

$$
y \in \partial^{\leq} f(x) \Longleftrightarrow(\forall w \in\{f \leq f(x)\} \quad f(w) \geq f(x)+\langle y, w-x\rangle) .
$$

Question. Would it be of interest to develop duality theories using new classes of elementary functions such as pseudo-affine or quasi-affine functions? 


\section{Continuity of Subdifferentials}

One has to face difficulties in devising calculus rules for the subdifferentials of quasiconvex analysis (see [238]). It is only with special classes of quasiconvex functions that one may expect to get useful rules. Let us consider for example, the class of functions which can be written under the form $h \circ g$, where $h$ is a given increasing function from some interval $I$ of $\mathbb{R}$ and $g$ belongs to the class of convex functions on some open convex subset $W$ of a n.v.s. $X$ taking their values in $T$. For instance, for $h:=\log , T$ being the set of positive real numbers, one obtains an important class. In such a case, one may expect to use the rules of convex analysis; in particular, for $f=\max _{i \in I} f_{i}$, where $I$ is a finite set and $f_{i}:=h \circ g_{i}$ with $g_{i}$ convex and $h$ increasing as above, one can compute the Greenberg-Pierskalla subdifferential of $f$.

In the present section we rather focus our attention to continuity properties of subdifferentials.It is well known that the subdifferential of a convex continuous function enjoys automatic semicontinuity properties. One may wonder whether such a fact remains valid for subdifferentials adapted to quasiconvex functions or whether it enables to define an interesting subclass of the class of quasiconvex functions.

Proposition 26. The subdifferential of a continuous convex function on an open convex subset $W$ of a n.v.s. $X$ is norm-to-weak ${ }^{*}$ upper semicontinuous. The lower (or Plastria) subdifferential of a continuous quasiconvex function is closed from the strong topology on $X$ to the bounded weak topology on $X^{*}$.

Proof. The first assertion is well known (see [242, Prop. 2.5, p. 19] for instance). In fact, for any continuous convex function $f: W \rightarrow \mathbb{R}$, the multimap $F:=\partial f: W \rightrightarrows X^{*}$ is scalarly upper semicontinuous in the sense that for any $x \in W, u \in X, r>\sigma_{F(x)}(u):=\sup \left\{\left\langle x^{*}, u\right\rangle: x^{*} \in F(x)\right\}$, one has $r>\sigma_{F(v)}(u)$ for $v$ in some neighborhood of $x$ (observe that there exists some $t>0$ such that $(1 / t)(f(x+t u)-f(x))<r$ and use the continuity of $f$ ). Let us prove the announced closedness of the Plastria subdifferential of $f$. Suppose on the contrary that there exist $\left(x, x^{*}\right) \in\left(W \times X^{*}\right) \backslash \partial^{<} f$ and a net $\left(\left(x_{i}, x_{i}^{*}\right)\right)_{i \in I}$ in the graph of $\partial^{<} f$ such that $\left(\left\|x_{i}-x\right\|\right) \rightarrow 0,\left(x_{i}^{*}\right)_{i \in I}$ is bounded and $\left(x_{i}^{*}\right) \rightarrow x^{*}$ weak*. Since $x^{*} \notin \partial^{<} f(x)$ there exists some $w \in W$ with $f(w)<f(x)$ such that $f(w)-f(x)<\left\langle x^{*}, w-x\right\rangle$. Then, for $i \in I$ larger than some $k \in I$ we have $f(w)<f\left(x_{i}\right)$ and $f(w)-f\left(x_{i}\right)<\left\langle x_{i}^{*}, w-x_{i}\right\rangle$, a contradiction with $x_{i}^{*} \in \partial^{<} f\left(x_{i}\right)$.

Example. Because the lower subdifferential is unbounded, even if the function is Lipschitzian and convex, closedness does not imply norm to weak* upper semicontinuity. As an example, consider the function $f$ on an Euclidean space $X$ (identified with its dual space) given by $f(x):=\|x\|$. Then for $x \in X \backslash\{0\}$ one has $\partial^{<} f(x)=[1,+\infty) x /\|x\|$, hence $\partial^{<} f(\cdot)$ is not upper semicontinuous at $x$.

Example. The Gutiérrez subdifferential of the function $f: x \rightarrow x_{-}:=\min (x, 0)$ is not graph-closed: for any sequence $\left(x_{n}\right) \rightarrow 0_{-}$one has $\partial \leq f\left(x_{n}\right)=[1,+\infty)$, but $\partial \leq f(0)=\varnothing$.

Another positive result can be given for the subdifferential considered in Section 5 .

Proposition 27. The subdifferential $\partial^{c_{\wedge}}$ of a continuous function is closed from the strong topology on $X$ to the bounded weak* topology on $X^{*}$.

Proof. Let $\left(\left(x_{i}, x_{i}^{*}\right)\right)_{i \in I}$ be a net in the graph of $\partial^{c_{\wedge}} f$ such that $\left(\left\|x_{i}-x\right\|\right) \rightarrow 0,\left(x_{i}^{*}\right)_{i \in I}$ is bounded and $\left(x_{i}^{*}\right) \rightarrow x^{*}$ weak $^{*}$. Since we have $\left\langle x_{i}^{*}, x_{i}\right\rangle \geq 1$ for all $i \in I$, we get $\left\langle x^{*}, x\right\rangle \geq 1$. Given $w \in\left\{x^{*} \geq 1\right\}$, we can find a net $\left(w_{i}\right)_{i \in I} \rightarrow w$ such that $w_{i} \in\left\{x_{i}^{*} \geq 1\right\}$ : it suffices to take $w_{i}:=w+t_{i} x$, with $t_{i}:=\left\langle x^{*}-x_{i}^{*}, w\right\rangle /\left\langle x_{i}^{*}, x\right\rangle$. Since, by definition of $\partial^{c_{\wedge}} f$, we have $f\left(w_{i}\right) \geq f\left(x_{i}\right)$ for all $i \in I$, we get $f(w) \geq f(x), f$ being continuous. Thus $x^{*} \in \partial^{c \wedge} f(x)$.

\section{Some SPECIAL ClASSES OF QUASICONVEX FUnCTIONS}

We believe that it is important to delineate nice classes of quasiconvex functions which are well structured. In particular, we are interested in stability properties of such classes for usual operations. Since addition of functions is not of interest for quasiconvex functions, we restrict our attention to supremum, composition with a nondecreasing function and sublevel convolution, the sublevel convolution of $g, h: X \rightarrow \overline{\mathbb{R}}$ being the function $g \diamond h$ defined by

$$
(g \diamond h)(x):=\inf \{g(u) \vee h(v): u, v \in X, u+v=x\} \quad x \in X
$$


We observe that $f:=g \diamond h$ is quasiconvex when $g$ and $h$ are quasiconvex since for every $r \in \mathbb{R}$

$$
S_{f}^{<}(r):=\{x \in X: f(x)<r\}=S_{g}^{<}(r)+S_{h}^{<}(r)
$$

Noting that the l.s.c. hull $\bar{f}$ of a quasiconvex function $f$ being still quasiconvex, we may also introduce the operation $\bar{\nabla}$ given by $g \bar{\nabla} h:=\overline{g \diamond h}$.

Proposition 28. The class of radiant functions is stable under suprema, sublevel convolution and reparameterization in the following sense: if $g: X \rightarrow \mathbb{R}$ is radiant and if $h: \mathbb{R} \rightarrow \mathbb{R}$ is l.s.c. and nondecreasing, then $f:=h \circ g$ is radiant. If moreover $g$ is l.s.c. and radiant, then $f:=h \circ g$ is l.s.c. and radiant.

Proof. The first two assertions are obvious. Let $f:=h \circ g$, where $g$ is radiant and $h$ is l.s.c. and nondecreasing. Given $r \in \mathbb{R}$, let $s:=\sup \{q \in \mathbb{R}: h(q) \leq r\}=\inf \{t \in \mathbb{R}: h(t)>r\}$. Then

$$
S_{f}(r)=S_{g}(s)
$$

In fact, for $x \in S_{f}(r)$ we have $q:=g(x) \leq s$ since $h(q) \leq r$. Conversely, if $x \in S_{g}(s)$ we cannot have $f(x)>r$ since otherwise we would have $h(q)>r$ for $q:=g(x)$, hence, by lower semicontinuity of $h$, there would exist some $p<q$ such that $h\left(q^{\prime}\right)>r$ for $q^{\prime} \in[p, q]$ and we would get $s \leq p<q=g(x)$, a contradiction with $x \in S_{g}(r)$. Relation (11) shows that $f$ is quasiconvex (resp. radiant) whenever $g$ is quasiconvex (resp. radiant). It also shows that $f$ is l.s.c. when $g$ is l.s.c.

Now let us turn to the important class of truncavex functions, a function being called a truncavex function if it is the supremum of a family of truncated continuous affine functions, i.e. a supremum of a family of functions of the form $a(\cdot) \wedge q$ where $a(\cdot)$ is a continuous affine function on $X$ and $q$ is a constant. This class of functions has interesting duality properties (see [181], [230]); it also plays some role for the study of Hamilton-Jacobi equations (see [1], [27], [28], [233]). Let us note that this class of functions is stable by truncation since for any families $\left(a_{i}\right)_{i \in I},\left(q_{i}\right)_{i \in I}$ of affine functions and real numbers, and for any $r \in \mathbb{R}$ one has

$$
\left(\sup _{i \in I}\left(a_{i} \wedge q_{i}\right)\right) \wedge r=\sup _{i \in I}\left(a_{i} \wedge q_{i} \wedge r\right) .
$$

Proposition 29. The class of truncavex functions is stable under suprema and is contained in the class of l.s.c. quasiconvex functions. If $g: X \rightarrow \mathbb{R}$ is truncavex and if $h: \mathbb{R} \rightarrow \mathbb{R}$ is l.s.c., nondecreasing and truncavex, then $f:=h \circ g$ is truncavex.

Proof. Stability by suprema is obvious. Let $f:=h \circ g$, with $g$ truncavex and $h: \mathbb{R} \rightarrow \mathbb{R}$ l.s.c., nondecreasing and truncavex. Using the characterization of [181, Prop. 4.2], in order to prove that $f$ is truncavex, it suffices to show that for every $r<\sup f$ there exists a continuous affine function $a$ minorizing $f$ on $S_{f}(r)$. Since $r<\sup f$, one also has $r<\sup h$ so that there exists a continuous affine function $c \leq h$ on $S_{h}(r)$. Let $s:=\sup \{q \in \mathbb{R}: h(q) \leq r\}$. Then $h(s) \leq r$ since $h$ is nondecreasing and l.s.c.. The preceding proof has shown that $S_{g}(s)=S_{f}(r) \neq X$, so that there exists some $\bar{x} \in X$ with $g(\bar{x})>s$. Since $g$ is truncavex, there exists a continuous affine function $b$ such that $b \leq g$ on $S_{g}(s)$. Now, for $x \in S_{g}(s)$, we have $h(g(x)) \leq r$, hence $g(x) \leq s$ by definition of $s$ and so $b(x) \leq s$; thus $h(b(x)) \leq r$ and $b(x) \in S_{h}(r)$. Therefore $c(b(x)) \leq h(b(x)) \leq h(g(x))$ and $a:=c \circ b$ is continuous affine and minorizes $f$ on $S_{f}(r)$.

Question. Is the class of truncavex functions stable by sublevel convolution? The following lemma shows that the case the sublevel convolution takes the value $-\infty$ is not excluded.

Lemma 30. Let $g:=b-\beta, h:=c-\gamma$ be two continuous affine functions on the n.v.s. $X$, with $b, c \in X^{*}$, $\beta, \gamma \in \mathbb{R}$. Then $f:=g \diamond h$ is also a continuous affine function or is identically $-\infty$.

Proof. If $b=0, c=0$ one has $f=(-\beta) \vee(-\gamma)$. If $b=0, c \neq 0$, then one has $f=-\beta$; similarly, when $b \neq 0, c=0$ one has $f=-\gamma$. Thus, we suppose $b \neq 0, c \neq 0$. Let us first suppose there exists some $\lambda>0$ such that $c=\lambda b$. Now, let us observe that for a non null linear form $b$ and $\alpha, \omega \in \mathbb{R}$ one has $b(x)<\alpha+\omega$ if, and 
only if, $x=u+v$ with $b(u)<\alpha, b(v)<\omega$ : it suffices to take $u:=(1 / 2) x+\mu e, v=(1 / 2) x-\mu e$ with $e \in b^{-1}(1)$, $\mu \in(-\omega+(1 / 2) b(x), \alpha-(1 / 2) b(x))$. Then, for any $r \in \mathbb{R}$ and $x \in X$, we have

$$
\begin{aligned}
f(x)<r & \Leftrightarrow \exists u, v \in X, u+v=x, b(u)-\beta<r, \lambda b(v)-\gamma<r \\
& \Leftrightarrow \exists u, v \in X, u+v=x, b(u)<\beta+r, b(v)<\lambda^{-1}(\gamma+r) \\
& \Leftrightarrow b(x)<\beta+r+\lambda^{-1}(\gamma+r) \\
& \Leftrightarrow \lambda(\lambda+1)^{-1} b(x)-(\lambda+1)^{-1}(\lambda \beta+\gamma)<r .
\end{aligned}
$$

It ensues that $f(x)=\lambda(\lambda+1)^{-1} b(x)-(\lambda+1)^{-1}(\lambda \beta+\gamma)$ for all $x \in X$ and $f$ is affine.

Now let us consider the case there is no $\lambda>0$ such that $c=\lambda b$. Since $c \neq 0$, there is no $\lambda \geq 0$ such that $c=\lambda b$. Then, by the Farkas lemma, the inequality $b(x) \geq 0$ does not imply the inequality $c(x) \geq 0$. Thus, there exists some $\bar{x} \in X$ such that $b(\bar{x}) \geq 0$ and $c(\bar{x})<0$. Changing $\bar{x}$ into $\bar{x}+r e$, with $b(e)>0, r>0$ small enough, we may suppose $b(\bar{x})>0$ and $c(\bar{x})<0$. Then, for $x \in X$, taking $u:=x-t \bar{x}, v:=t \bar{x}$ with $t>0$ large, we see that $(g \diamond h)(x)=-\infty$.

Question. When is a truncavex function $\partial$-pseudoconvex?

Now let us consider the class of transconvex functions. Here a function $f$ on an open convex subset $W$ of a n.v.s. $X$ is said to be transconvex if there exist a continuous convex function $g: W \rightarrow \mathbb{R}$ and a differentiable nondecreasing function $h: I \rightarrow \mathbb{R}$ on some interval $I$ of $\mathbb{R}$ such that $g(W) \subset I$ and $f=h \circ g$. If $h$ just has a left derivative at each point, we say that $f$ is left transconvex. This definition slightly extends the class considered in [214] (where $h$ is required to be differentiable everywhere). We are not interested in these classes for their stability properties but for their links with a subdifferential introduced in [214] which is local and not global. The construction is as follows. Given a function $f$, a point $x$ at which $f$ is finite and a l.s.c. approximation $f_{x}$ of $f$ at $x$ in the sense adopted for relation (3), one sets

$$
\partial f(x):=\partial^{<} f_{x}(0)
$$

Setting $D:=\left\{f_{x}<0\right\} \cup\{0\}$ and introducing $f_{x}^{<}: X \rightarrow \overline{\mathbb{R}}$ by $f_{x}^{<}(u):=f_{x}(u)$ for $u \in \operatorname{cl}(D), f_{x}^{<}(u):=+\infty$ for $u \in X \backslash \operatorname{cl}(D)$, we see that $\partial f(x)$ is also the Fenchel subdifferential of $f_{x}^{<}$at 0 :

$$
\partial f(x)=\left\{x^{*} \in X^{*}: x^{*} \leq f_{x}^{<}\right\}
$$

This observation made in [214, Lemma 2.1] stems from the fact that $f_{x}$ is null on $\operatorname{cl}(D) \backslash D$. Since $f_{x}^{<}$is l.s.c. as $f_{x}$ is l.s.c., $\partial f(x)$ is nonempty whenever $f_{x}^{<}$is sublinear. In turn, this occurs when $f_{x}$ is quasiconvex and $f_{x}(0)=0$ (see [56], [211, Thm 1]). In general the contingent derivative $f^{\prime}(x, \cdot)$ does not satisfy this property. But its close variant, the incident (or adjacent) derivative $f^{i}(x, \cdot)$ given by

$$
\begin{aligned}
f^{i}(x, u) & :=\operatorname{epi}-\limsup _{t \searrow 0} \frac{1}{t}(f(x+t \cdot)-f(x))(u) \\
& :=\sup _{r>0} \limsup _{t \searrow 0} \inf _{v \in B(u, r)} \frac{1}{t}(f(x+t v)-f(x)) .
\end{aligned}
$$

does satisfy it when $f$ is quasiconvex and $f^{i}(x, 0) \neq-\infty$.

The following result enhances the interest of (left) transconvex functions.

Proposition 31. Let $f:=h \circ g$ be a left transconvex function. Then $f^{i}(x, \cdot)$ and $f_{x}:=f^{\prime}(x, \cdot)$ coincide on $\operatorname{cl}(D)$ for $D:=\left[f_{x}<0\right] \cup\{0\}$. Moreover, $f_{x}^{<}$defined as above is sublinear and $\partial f_{x}^{<}(0)$ is nonempty. In fact, $\partial f_{x}^{<}(0)=h_{-}^{\prime}(r) \partial g(x)+D^{0}$, where $h_{-}^{\prime}(r)$ is the left derivative of $h$ at $r:=g(x)$ and $D^{0}$ is the polar cone of $D$. If $f$ is transconvex, then $f$ has a directional derivative at $x$ which is sublinear and continuous: $f^{\prime}(x, \cdot)=h^{\prime}(g(x)) g^{\prime}(x, \cdot)$ and the contingent subdifferential of $f$ at $x$ is $h^{\prime}(g(x)) \partial g(x)$. 
Proof. Let us first consider the case of a left transconvex function $f=h \circ g$ as above. Let $u \in D$ i.e. be such that $f^{\prime}(x, u)<0$ and let $\left(t_{n}\right) \rightarrow 0_{+},\left(u_{n}\right) \rightarrow u$ be sequences such that $f^{\prime}(x, u)=\lim _{n}\left(1 / t_{n}\right)\left(f\left(x+t_{n} u_{n}\right)-f(x)\right)$. Since $g$ is convex continuous, $g$ has a directional derivative and

$$
p_{n}:=\frac{1}{t_{n}}\left(g\left(x+t_{n} u_{n}\right)-g(x)\right) \rightarrow p:=g^{\prime}(x, u) .
$$

For $n$ large enough we have $g\left(x+t_{n} u_{n}\right)<r:=g(x)$ since otherwise we would have $f^{\prime}(x, u) \geq 0, h$ being nondecreasing. Thus, $p_{n}<0$ for $n$ large and

$$
h\left(r+t_{n} p_{n}\right)=: h(r)+t_{n} p_{n} q_{n} \text { where }\left(q_{n}\right) \rightarrow h_{-}^{\prime}(r)
$$

It follows that $f^{\prime}(x, u)=\lim _{n}\left(1 / t_{n}\right)\left(h\left(r+t_{n} p_{n}\right)-h(r)\right)=\lim _{n} p_{n} q_{n}=g^{\prime}(x, u) h_{-}^{\prime}(r)$. Thus $g^{\prime}(x, u)<0$, $h_{-}^{\prime}(r)>0$ and for any other sequences $\left(t_{n}^{\prime}\right) \rightarrow 0_{+},\left(u_{n}^{\prime}\right) \rightarrow u$ we have

$$
\begin{aligned}
& p_{n}^{\prime}:=\frac{1}{t_{n}^{\prime}}\left(g\left(x+t_{n}^{\prime} u_{n}^{\prime}\right)-g(x)\right) \rightarrow p:=g^{\prime}(x, u), \\
& q_{n}^{\prime}:=\frac{1}{t_{n}^{\prime}}\left(h\left(r+t_{n}^{\prime} p_{n}^{\prime}\right)-h(r)\right) \rightarrow h_{-}^{\prime}(r),
\end{aligned}
$$

hence $\left(1 / t_{n}^{\prime}\right)\left(f\left(x+t_{n}^{\prime} u_{n}^{\prime}\right)-f(x)\right) \rightarrow g^{\prime}(x, u) h_{-}^{\prime}(r)$. Thus $f$ has a derivative in the direction $u$. In particular $f^{\prime}(x, u)$ coincides with the epiderivative $f^{i}(x, u)$. For $u \in \operatorname{cl}(D) \backslash D$ we have

$$
0 \leq f^{\prime}(x, u) \leq f^{i}(x, u) \leq 0
$$

by definition of $D$ and by the lower semicontinuity of $f^{i}(x, \cdot)$. Thus $f^{\prime}(x, u)=f^{i}(x, u)=0$ and $f^{\prime}(x, \cdot)$ and $f^{i}(x, \cdot)$ coincide on $\operatorname{cl}(D)$. Since $f^{i}(x, \cdot)$ is quasiconvex, the function $f_{x}^{<}$given by $f_{x}^{<}(u)=f^{\prime}(x, u)$ for $u \in \operatorname{cl}(D)$, $f_{x}^{<}(u):=+\infty$ for $u \in X \backslash \operatorname{cl}(D)$ is sublinear and l.s.c. $([58],[213])$. Since $f_{x}^{<}=h_{-}^{\prime}(r) g^{\prime}(x, \cdot)+\iota_{\operatorname{cl}(D)}$, and since $g$ is continuous at $x$, by the familiar sum rule of convex analysis, we get $\partial f_{x}^{<}(0)=h_{-}^{\prime}(r) \partial g(x)+\operatorname{cl}(D)^{0}=$ $h_{-}^{\prime}(r) \partial g(x)+D^{0}$.

When $f$ is transconvex, the computation of the directional derivative of $f$ is simpler and the formula $f^{\prime}(x, \cdot)=$ $h^{\prime}(g(x)) g^{\prime}(x, \cdot)$ immediately yields the contingent subdifferential since $h^{\prime}(g(x)) \geq 0$ and $\partial g(x)$ is nonempty.

Question. Can one give conditions yielding the Plastria subdifferential of a transconvex function?

Partial results have been given in [236, Prop. 3.5]. We quote one of them, with a slight adjustment.

Proposition 32. Let $g: X \rightarrow \mathbb{R}_{\infty}, h: \mathbb{R}_{\infty} \rightarrow \mathbb{R}_{\infty}$ be nondecreasing with $h(+\infty)=+\infty$. Let $x \in X$ be such that $r:=g(x) \in \mathbb{R}$ and $h(r) \in \mathbb{R}$. Then $\partial^{<} h(r) \partial^{<} g(x) \subset \partial^{<}(h \circ g)(x)$. Suppose $g$ is l.s.c., sublinear, with $g(x)>\inf g$ and $r$ is not a local minimizer of $h$. Then equality holds if either $g(x) \leq 0$ or $g(x)>0$ and $h(t)=h(0)$ for $t \leq 0$.

\section{D.C. FUNCTIONS AND QUASICONVEX FUNCTIONS}

Recall that a function $f: X \rightarrow \mathbb{R}_{\infty}$ is said to be a d.c. function if there are two convex functions $g, h: X \rightarrow$ $\mathbb{R}_{\infty}$ such that $f=g-h$. Such functions have been extensively studied (see [2], [43], [84], [85], [292], [293], [305], [307] for a survey and references). They occur frequently ( [122], [224]). It has been proved by Asplund that the square of the distance function to a nonempty closed subset of a Hilbert space is a d.c. function. It is also the case locally for the distance function itself on the complement of the set ([35, p. 214]).

Question. Given a d.c. function $f=g-h$, and a subdifferential $\partial$, under what assumptions is it $\partial$-invex, quasiconvex or $\partial$-pseudoconvex?

A similar question arises when $f$ is tangentially d.s. in the sense of [43], i.e. when for every $x \in \operatorname{dom} f$ the contingent derivative $f^{\prime}(x, \cdot)$ is the difference of two sublinear functions. 
Let us give some elements for an answer. We need the concept of gap-continuity of a set-valued map introduced in [222]. A multimap $F: X \rightrightarrows Y$ between two n.v.s. is said to be gap-continuous at $x \in X$ if

$$
\operatorname{gap}(F(w), F(x)) \rightarrow 0 \text { as } w \rightarrow x
$$

where, for two subsets $A, B$ of $Y, \operatorname{gap}(A, B):=\inf \{d(a, b): a \in A, b \in B\}$. We also set

$$
A \boxminus B:=\{y \in Y: B+y \subset A\} .
$$

The Fréchet subdifferential of a function $f$ is denoted by $\partial^{-}$. For $x \in \operatorname{dom} f$, it is defined by

$$
x^{*} \in \partial^{-} f(x) \Leftrightarrow f(x+w)-f(x)-\left\langle x^{*}, w\right\rangle \geq o(\|w\|)
$$

Proposition 33. Let $g: W \rightarrow \mathbb{R}_{\infty}, h: W \rightarrow \mathbb{R}$ be convex functions on an open convex subset $W$ of a n.v.s. $X$ and let $f:=g-h$.

(a) If the inclusion $\partial h(x) \subset \partial g(x)$ implies that $g(w)-g(x) \geq h(w)-h(x)$ for all $w \in W$, then $f$ is $\partial^{-}$-invex. If $\partial h$ is gap-continuous at each point of $W$ and if $f$ is $\partial^{-}$-invex, then this implication holds.

(b) In order that $f$ be $\partial^{-}$-quasiconvex it suffices that for every $x, y \in X, x^{*} \in \partial g(x) \boxminus \partial h(x)$ the inequality $f(y) \geq f(x)$ holds whenever $\left\langle x^{*}, y-x\right\rangle>0$. If $\partial h$ is gap-continuous at each point of $X$, this condition is necessary.

(c) In order that $f$ be $\partial^{-}$-pseudoconvex it suffices that for every $x, y \in X, x^{*} \in \partial g(x) \boxminus \partial h(x)$ the inequality $f(y) \geq f(x)$ holds whenever $\left\langle x^{*}, y-x\right\rangle \geq 0$. If $\partial h$ is gap-continuous at each point of $X$, this condition is necessary.

Proof. (a) Suppose $\partial h(x) \subset \partial g(x)$ implies that $g(w)-g(x) \geq h(w)-h(x)$ for all $w \in W$. Since the implication

$$
0 \in \partial^{-} f(x) \Rightarrow \partial h(x) \subset \partial g(x)
$$

always holds, as observed in [212, Prop. 2.2], when $0 \in \partial^{-} f(x)$, for all $w \in W$, we obtain $g(w)-g(x) \geq$ $h(w)-h(x)$, or $f(w) \geq f(x): f$ is $\partial^{-}$-invex. When $\partial h$ is gap-continuous at each point $x$ of $X$, by [2] one has

$$
\partial^{-} f(x)=\partial g(x) \boxminus \partial h(x):=\left\{x^{*} \in X^{*}: \partial h(x)+x^{*} \subset \partial g(x)\right\},
$$

so that $0 \in \partial^{-} f(x)$ if, and only if, $\partial h(x) \subset \partial g(x)$. Thus, when this inclusion holds and $f$ is $\partial^{-}$-invex, we get $f(w) \geq f(x)$ for all $w \in W$, or $g(w)-g(x) \geq h(w)-h(x)$.

Assertion (b) (c) are immediate consequences of the inclusion $\partial^{-} f(x) \subset \partial g(x) \boxminus \partial h(x)$, with equality when $\partial h$ is gap-continuous.

\section{REFERENCES}

[1] O. Alvarez, E.N. Barron and H. Ishii, Hopf-Lax formulas for semicontinuous data, Indiana Univ. Math. J. 48, No.3, 993-1035 (1999).

[2] T. Amahroq, J.-P. Penot and A. Syam, On the subdifferentiability of the difference of two functions and local minimization, preprint, Sept. 2005.

[3] C. Amara, Directions de majoration d'une fonction quasiconvexe et applications. (Upper bound directions of a quasi-convex function and applications), Serdica Math. J. 24, No.3-4 (1998), 289-306.

[4] C. Amara and M. Volle, More on quasiconvex duality (Compléments de dualité quasi convexe), Ann. Sci. Math. Qué. 23, No.2 (1999), 119-137.

[5] K.J. Arrow and A.C. Enthoven, Quasi-concave programming, Econometrica 29, 779-800 (1961).

[6] M. Atteia, Analyse convexe projective, C. R. Acad. Sci., Paris, Sér. A 276, 795-798 \& 855-858 (1973).

[7] M. Atteia and A. El Qortobi, Quasi-convex duality, Optimization and optimal control, Proc. Conf., Oberwolfach 1980, Lect. Notes Control Inf. Sci. 30, 3-8 (1981).

[8] M. Atteia and A. El Qortobi, Hamiltoniens quasi-convexes quasi-concaves, RAIRO, Rech. Opér. 25, No.4, 425-438 (1991). 
[9] J.-P. Aubin, Mathematical methods of game and economic theory, Studies in Mathematics and its Applications, Vol. 7, North-Holland, Amsterdam (1979).

[10] J.-P. Aubin and H. Frankowska, Set-Valued Analysis, Birkhäuser, Basel, 1990.

[11] D. Aussel, Subdifferential properties of quasiconvex and pseudoconvex functions: unified approach, J. Optim. Th. Appl. 97 (1), 29-45 (1998).

[12] D. Aussel, J.N. Corvellec and M. Lassonde, Subdifferential characterization of quasiconvexity and convexity, J. Convex Anal. 1, No.2, 195-201 (1994)

[13] D. Aussel, J.N. Corvellec and M. Lassonde, Mean value property and subdifferential criteria for lower semicontinuous functions, Trans. Am. Math. Soc. 347, No.10, 4147-4161 (1995).

[14] D. Aussel and D. Daniilidis, Normal characterization of the main classes of quasiconvex functions, Set-Valued Anal. 8, No.3, 219-236 (2000).

[15] D. Aussel and D. Daniilidis, Normal cones to sublevel sets: An axiomatic approach. Applications in quasiconvexity and pseudoconvexity, in Generalized convexity and generalized monotonicity. Proceedings of the 6th international symposium, Samos, Greece, September 1999. N. Hadjisavvas, J.E. Martínez-Legaz and J.-P. Penot (eds.), Lect. Notes Econ. Math. Syst. 502, Springer, Berlin, 88-101 (2001).

[16] D. Aussel and A. Daniilidis, Normal characterization of the main classes of quasiconvex functions, Set-Valued Anal. 8, No.3, 219-236 (2000)

[17] D. Aussel, A. Daniilidis, and L. Thibault, Subsmooth sets: functional characterizations and related concepts, Trans. Amer. Math. Soc. 357, No.4 (2005), 1275-1301.

[18] D. Aussel and N. Hadjisavvas, Adjusted sublevel sets, normal operator and quasiconvex programming, preprint, Univ. Perpignan, (2004).

[19] M. Avriel, $r$-convex functions, Math. Program. 2, 309-323 (1972).

[20] M. Avriel, Nonlinear Programming. Analysis and Methods. Prentice-Hall, Englewood Cliffs, N.J. (1976). Reprint, Dover, Mineola, N.Y. (2003)

[21] M. Avriel, W.E. Diewert, S. Schaible and I. Zang, Generalized Concavity, Plenum Press, New York and London 1988.

[22] M. Avriel, W.E. Diewert, S. Schaible and W.T. Ziemba, Introduction to concave and generalized concave functions, in Generalized concavity in optimization and economics, Proc. NATO Adv. Study Inst., Vancouver/Can. 1980, 21-50 (1981).

[23] D. Azé and M. Volle, A stability result in quasi-convex programming, J. Optim. Theory Appl. 67, No.1 (1990), 175-184.

[24] M. Avriel and S. Schaible, Second order characterizations of pseudoconvex functions. Math. Program. 14, 170-185 (1978).

[25] M. Bachir, A. Daniilidis, and J.-P. Penot, Lower subdifferentiability and integration, Set-Valued Anal. 10, No.1, 89-108 (2002).

[26] Barbara, A. and Crouzeix, J.-P., Concave gauge functions and applications, Z. Oper. Res. 40, No.1, 43-74 (1994).

[27] E.N. Barron, Viscosity solutions and analysis in $L_{\infty}$, in "Nonlinear Analysis, Differential Equations and Control", F.H. Clarke and R.J. Stern editors, NATO Science Series 528, Kluwer, Dordrecht (1999), 1-60.

[28] E.N. Barron, R. Jensen and W. Liu, Hopf-lax type formula for $u_{t}+H(u, D u)=0$. II. Commun. Partial Differ. Equations 22 , No.7-8, 1141-1160 (1997).

[29] A. Ben Israel and B. Mond, What is invexity?, J. Austral. Math. Soc. B 28, 1-9 (1982).

[30] M. Bianchi, Generalized quasimonotonicity and strong pseudomonotonicity of bifunctions, Optimization 36 (1) (1996) 1-10.

[31] M. Bianchi and S. Schaible, An extension of pseudolinear functions and variational inequality problems, J. Optimization Theory Appl. 104, No.1, 59-71 (2000).

[32] M. Bianchi, N. Hadjisavvas and S. Schaible, On pseudomonotone maps $T$ for which $-T$ is also pseudomonotone, J. Convex Anal. 10, No.1, 149-168 (2003).

[33] J. Borde and J.-P. Crouzeix, Continuity properties of the normal cone to the level sets of a quasiconvex function, J. Optimization Theory Appl. 66, No.3, 415-429 (1990).

[34] J. Borwein, Fractional programming without differentiability, Math. Programming 11 (1976), 283-290.

[35] J.M. Borwein and Q.J. Zhu, Techniques of Variational Analysis, CMS Books in Maths Springer, New York (2005).

[36] A. Cambini, E. Castagnoli, L. Martein, P. Mazzoleni, S. Schaible (eds), Generalized Convexity and fractional programming with economic applications, Proc. Pisa, 1988, Lecture Notes in Economics and Math. Systems 345, Springer Verlag, Berlin, 1990.

[37] A. Cambini, J.-P. Crouzeix and L. Martein, On the pseudoconvexity of a quadratic fractional function, Optimization 51 (2002), 667-687.

[38] A. Cambini and L. Martein, Generalized convexity and optimality conditions in scalar and vector optimization, in N. Hadjisavvas, S. Komlósi and S. Schaible, Handbook of generalized convexity and generalized monotonicity, Springer, New York (2005), 151-193.

[39] A. Cambini and L. Martein and S. Schaible, Pseudoconvexity, pseudomonotonicity and the generalized Charnes-Cooper transformation. Pac. J. Optim. 1 (2005), no. 2, 265-275.

[40] A. Cambini and L. Martein and S. Schaible, On the pseudoconvexity of the sum of two linear fractional functions. in Generalized convexity, generalized monotonicity and applications, Nonconvex Optim. Appl., 77, Springer, New York, (2005), 161-172. 
[41] R. Cambini and L. Carosi, On generalized linearity of quadratic fractional functions, J. Global Optim. 30 (2-3) (2004), $235-251$.

[42] R. Cambini and L. Carosi and S. Schaible, Duality in fractional programming problems with set constraints. Generalized convexity, generalized monotonicity and applications, 147-159, Nonconvex Optim. Appl., 77, Springer, New York, 2005.

[43] E. Caprari and J.-P. Penot, Tangentially d.-s. functions, Optimization 56 (1), (2007), 25-38.

[44] D. Carrasco-Olivera, F. Flores-Bazán, On the representation of approximate subdifferentials for a class of generalized convex functions, Set-Valued Anal. 13 (2), 151-166.

[45] E. Castagnoli, P. Mazzoleni, About derivatives of some generalized concave functions, J. Inf. Optimization Sci. 10, No.1 (1989), 53-64.

[46] M. Castellani, Nonsmooth invex functions and sufficient optimality conditions, J. Math. Anal. Appl. 255 (1) (2001), 319-332.

[47] F.H. Clarke, Optimization and Nonsmooth Analysis, Wiley, New-York, 1983.

[48] F.H. Clarke, Yu.S. Ledyaev, R.J. Stern \& P.R. Wolenski, Nonsmooth Analysis and Control Theory, Springer, New York (1998).

[49] Colombo, G., and Goncharov, V., Variational inequalities and regularity properties of closed sets in Hilbert spaces, J. Convex Anal. 8 (2001), 197-221..

[50] R. Correa, A. Jofre and L. Thibault, Characterization of lower semicontinuous convex functions, Proc. Amer. Math. Soc. 116 (1992), 67-72.

[51] R. Correa, A. Jofre and L. Thibault, Subdifferential monotonicity as characterization of convex functions, Numer. Funct. Anal. Optim. 15 (5\&6) (1994) 531-535.

[52] R. Correa, A. Jofre and L. Thibault, Subdifferential characterization of convexity, in Recent advances in nonsmooth optimization, 18-23, World Scientific, River Edge, NJ, 1998.

[53] J.-P. Crouzeix, Contribution à l'étude des fonctions quasi-convexes, Thèse d'Etat, Univ. de Clermont II, 1977.

[54] J.-P. Crouzeix, Condition for convexity of a quasiconvex function, Math. Oper. Res. 5 (1) (1980), $120-125$.

[55] J.-P. Crouzeix, Some differentiability properties of quasiconvex functions on $R^{n}$, in Optimization and optimal control, Proceedings Conference Oberwolfach 1980, A. Auslender, W. Oettli and J. Stoer, eds. Lecture Notes in Control and Information Sci. 30, Springer-Verlag (1981), 89-104.

[56] J.-P. Crouzeix, Continuity and differentiability properties of quasiconvex functions on $\mathbb{R}^{n}$, in Generalized concavity in optimization and economics, Proc. NATO Adv. Study Inst., Vancouver, 1980, 109-130 (1981).

[57] J.-P. Crouzeix, A review of continuity and differentiability properties of quasiconvex functions on $\mathbb{R}^{n}$, in Convex analysis and optimization, Colloq. London 1980, Res. Notes Math. 57, 18-34 (1982).

[58] J.-P. Crouzeix, About differentiability of order one of quasiconvex functions on $\mathbb{R}^{n}$, J. Optim. Theory Appl. 36, 367-385 $(1982)$.

[59] J.-P. Crouzeix, Pseudomonotone variational inequality problems: existence of solutions, Math. Programming 78 (1997), 305-314.

[60] J.-P. Crouzeix, La convexité généralisée en économie mathématique (Generalized convexity in mathematical economics), ESAIM, Proc. 13, 31-40, (2003).

[61] J.-P. Crouzeix, Criteria for generalized convexity and generalized monotonicity in the differentiable case, in N. Hadjisavvas, S. Komlósi and S. Schaible, Handbook of generalized convexity and generalized monotonicity, Springer, New York (2005), 89-119.

[62] J.-P. Crouzeix, Continuity and differentiability of quasiconvex functions, in N. Hadjisavvas, S. Komlósi and S. Schaible, Handbook of generalized convexity and generalized monotonicity, Springer, New York (2005), 121-149.

[63] J.-P. Crouzeix and J.A. Ferland, Criteria for quasi-convexity and pseudo-convexity: relationships and comparisons, Math. Programming 23 (1982), 193-205.

[64] J.-P. Crouzeix and J.A. Ferland, Criteria for differentiable generalized monotone maps, Math. Program. 75A, No.3, 399-406 (1996).

[65] J.-P. Crouzeix, J.A. Ferland and S. Schaible, Duality in generalized linear fractional programming, Math. Program. 27, 342-354 (1983).

[66] J.-P. Crouzeix, J.A. Ferland and S. Schaible, Generalized convexity on affine subspaces with an application to potential functions, Math. Program., Ser. A 56, No.2, 223-232 (1992).

[67] J.-P. Crouzeix, J.A. Ferland and C. Zalinescu, $\alpha$-convex and strong quasiconvexity, Math. Oper. Res. 22, No.4, 998-1022 (1997).

[68] J.-P. Crouzeix and A. Hassouni, Quasimonotonicity of separable operators and monotonicity indices, SIAM J. Optim. 4, No.3, 649-658 (1994).

[69] J.-P. Crouzeix and A. Hassouni, Generalized monotonicity of a separable product of operators: the multivalued case. SetValued Anal. 3 (1995), no. 4, 351-373.

[70] J.-P. Crouzeix, A. Hassouni, A. Lahlou, and S. Schaible, Positive subdefinite matrices, generalized monotonicity, and linear complementarity problems, SIAM J. Matrix Anal. Appl. 22, No.1, 66-85 (2000).

[71] J.-P. Crouzeix and T. Rapcsák, Integrability of pseudomonotone differentiable maps and the revealed preference problem, preprint, Nov. 2003. 
[72] A. Daniilidis and P. Georgiev, Approximate convexity and submonotonicity, J. Math. Anal. Appl. 291 (2004), $292-301$.

[73] A. Daniilidis, P. Georgiev and J.-P. Penot, Integration of multivalued operators and cyclic submonotonicity, Trans. Amer. Math. Soc. 355 (2003), 177-195.

[74] A. Daniilidis, N. Hadjisavvas, Characterization of nonsmooth semistrictly quasiconvex and strictly quasiconvex functions, J. Optim. Theory Appl. 102, No.3, 525-536 (1999).

[75] A. Daniilidis, N. Hadjisavvas, Coercivity conditions and variational inequalities, Math. Program. 86A, No.2, 433-438 (1999).

[76] A. Daniilidis, N. Hadjisavvas, On the subdifferentials of quasiconvex and pseudoconvex functions and cyclic monotonicity, J. Math. Anal. Appl. 237, No.1, 30-42 (1999).

[77] A. Daniilidis, N. Hadjisavvas, On generalized cyclically monotone operators and proper quasimonotonicity, Optim. 47, No.1-2, 123-135 (2000).

[78] A. Daniilidis, N. Hadjisavvas and J.-E. Martínez-Legaz, An appropriate subdifferential for quasiconvex functions, SIAM J. Optim. 12, No.2, 407-420 (2001).

[79] M. Degiovanni, A. Marino, M. Tosques, Evolution equations with lack of convexity, Nonlinear Anal. 9 (1985), $1401-1443$.

[80] R. Deville, A mean value theorem for nondifferentiable mappings, A mean value theorem for non differentiable mappings in Banach spaces, Serdica Math. J. 21, No.1, 59-66 (1995).

[81] R. Deville, G. Godefroy and V. Zizler, A smooth variational principle with applications to Hamilton-Jacobi equations in infinite dimensions, J. Funct. Anal. 111 (1993), 197-212.

[82] W.E. Diewert, Alternative characterizations of six kinds of quasiconcavity in the nondifferentiable case with applications to nonsmooth programming, in Generalized Concavity in Optimization and Economics, S. Schaible and W.T. Ziemba (eds.), Academic Press, New-York, 1981.

[83] W.E. Diewert, M. Avriel and I. Zang, Nine kinds of quasiconcavity and concavity, J. Econ. Theory 25, 397-420 (1981).

[84] N. Dinh, G. Vallet and T.T.A. Nghia, Farkas-type results and duality for DC programs with convex constraints, preprint, Intern. Univ. Ho Chi Minh City (2006).

[85] N. Dinh, T.T.A. Nghia and G. Vallet, A closedness condition and its application to DC programs with convex constraints, preprint, Univ. of Pau (2006).

[86] S. Dolecki and S. Kurcyusz, On $\Phi$-convexity in extremal problems, SIAM J. Control Optim. 16 (1978), 277-300.

[87] J. Dutta, S. Chandra, Convexifactors, generalized convexity and vector optimization, Optimization 53 (1) (2004), 77-94.

[88] J. Dutta, V. Vetrivel, S. Nanda, Semi-invex functions and their subdifferentials, Bull. Aust. Math. Soc. 56 (1997), 385-393.

[89] R. Ellaia and H. Hassouni, Characterization of nonsmooth functions through their generalized gradients, Optimization 22 (1991), 401-416.

[90] M. Fabian, Subdifferentials, local $\varepsilon$-supports and Asplund spaces, J. London Math. Soc.(2) 34 (1986), 568-576.

[91] M. Fabian, On classes of subdifferentiability spaces of Ioffe, Nonlinear Anal., Th. Meth. Appl. 12 (1) (1988), 63-74.

[92] M. Fabian, Subdifferentiability and trustworthiness in the light of a new variational principle of Borwein and Preiss, Acta Univ. Carolinae 30 (1989), 51-56.

[93] M. Fabian and N. V. Zivkov, A characterization of Asplund spaces with help of local $\varepsilon$-support of Ekeland-Lebourg, C.R. Acad. Bulgare Sci. 38 (1985), 671-674.

[94] K. Fan, Minimax theorems, Proc. Natl. Acad. Sci. USA 39, 42-47 (1953).

[95] K. Fan, On infinite systems of linear inequalities, J. Math. Anal. Appl. 21, 475-478 (1968).

[96] L. Fan, S. Liu, S. Gao, Generalized monotonicity and convexity of non-differentiable functions, J. Math. Anal. Appl. 279 (2003), 276-289.

[97] B. de Finetti, Sulle stratificazioni convesse, Ann. Mat. Pura Appl. 30 (1949), 173-183.

[98] F. Flores-Bazán and J.-E. Martínez-Legaz, Simplified global optimality conditions in generalized conjugation theory, in Generalized convexity, generalized monotonicity: recent results. Proceedings of the 5th symposium, Luminy, France, June 17-21, 1996. J.-P. Crouzeix et al. (eds.), Kluwer, Dordrecht (1998), 305-329.

[99] F. Flores-Bazán, On a notion of subdifferentiability for non-convex functions, Optimization 33, No.1 (1995), 1-8.

[100] F. Flores-Bazán, On minima of the difference of functions, J. Optim. Theory Appl. 93, No.3 (1997), 525-531.

[101] F. Flores-Bazán, Ideal, weakly efficient solutions for vector optimization problems, Math. Program. 93, No.3(A) (2002), 453-475.

[102] R. P. Gilbert, P.D. Panagiotopoulos and P. M. Pardalos (Editors), From convexity to nonconvexity, Nonconvex Optimization and its Applications, 55, Kluwer, Dordrecht, 2001.

[103] I. Ginchev and V. I. Ivanov, Second-order characterizations of convex and pseudoconvex functions. J. Appl. Anal. 9 (2003), no. 2, 261-273.

[104] G. Giorgi, A note on the relationships between convexity and invexity, J. Austral. Math. Soc. Ser. B 32 (1990), 97-99.

[105] G. Giorgi, On sufficient optimality conditions for a quasiconvex programming problem, J. Optim. Theory Appl. 81 (2) (1994), 401-405.

[106] G. Giorgi and S. Komlósi, Dini derivatives in optimization, I Riv. Mat. Sci. Econ. Soc. 15, No.1, 3-30 (1992), II idem 15, No.2, 3-24 (1992), III ibidem 18, No.1, 47-63 (1995).

[107] D. Goeleven, D. Motreanu and P. D. Panagiotopoulos, Semicoercive variational-hemivariational inequalities. Appl. Anal. 65 (1997), no. 1-2, 119-134. 
[108] H.J. Greenberg, W.P. Pierskalla, A review of quasi-convex functions, Oper. Res. 19 (1971), 1553-1570.

[109] Guerraggio, A., Molho, E., The origins of quasi-concavity: A development between mathematics and economics, Historia Math. 31 (1) (2004), 62-75

[110] S. Guillaume, Subdifferential evolution inclusion in nonconvex analysis, Positivity 4, No.4, 357-395 (2000).

[111] S. Guillaume, Time dependent subdifferential evolution equations in nonconvex analysis, Adv. Math. Sci. Appl. 10, No.2, 873-898 (2000).

[112] S. Guillaume, Duality and well behavior of nonconvex functions, J. Optim. Theory Appl. 114, No.3, 507-543 (2002).

[113] Haberl, J. Maximization of generalized convex functionals in locally convex spaces, J. Optim. Theory Appl. 121 (2) (2004), 327-359.

[114] N. Hadjisavvas, The use of subdifferentials for studying generalized convex functions, J. Statistics Management Sci. 5 (2002), $125-139$

[115] N. Hadjisavvas, Generalized convexity, generalized monotonicity and nonsmooth analysis, in N. Hadjisavvas, S. Komlósi and S. Schaible, Handbook of generalized convexity and generalized monotonicity, Springer, New York (2005), 465-499.

[116] N. Hadjisavvas, S. Komlósi and S. Schaible, Handbook of generalized convexity and generalized monotonicity, Springer, New York (2005).

[117] N. Hadjisavvas, J.-E. Martínez-Legaz and J.-P. Penot, Generalized convexity and generalized monotonicity. Proceedings of the 6th international symposium, Samos, Greece, September 1999, Lecture Notes in Econ. and Math. Systems \# 502, Springer, Berlin (2001).

[118] N. Hadjisavvas and S. Schaible, On strong pseudomonotonicity and (semi)strict quasimonotonicity, J. Optim. Theory Appl. 85, No.3, 741-742 (1995).

[119] N. Hadjisavvas and S. Schaible, Quasimonotone variational inequalities in Banach spaces, J. Optim. Th. Appl. 90 (1996), 95-111.

[120] N. Hadjisavvas and S. Schaible, Quasimonotonicity and pseudomonotonicity in variational inequalities and equilibrium problems, in Generalized convexity, generalized monotonicity: recent results. Proceedings of the 5th symposium, Luminy, France, June 17-21, 1996, J.-P. Crouzeix et al. (eds.), Nonconvex Optim. Appl. 27, Kluwer Dordrecht 257-275 (1998).

[121] M. A. Hanson, On sufficiency of the Kuhn-Tucker conditions, J. Math. Anal. Appl. 80, 545-550 (1981).

[122] Ph. Hartman, On functions representable as a difference of convex functions, Pac. J. Math. 9 (1959), $707-713$.

[123] A. Hassouni, Sous-différentiel des fonctions quasi-convexes", Thèse de troisième cycle, Université P. Sabatier, Toulouse (1983).

[124] A. Hassouni, Opérateurs quasimonotones; applications à certains problèmes variationnels, thesis, Univ. Toulouse (1993).

[125] A. Hassouni, Quasimonotone multifunctions; applications to optimality conditions in quasiconvex programming, Numer. Funct. Anal. Optimization 13, No.3-4, 267-275 (1992).

[126] A. Hassouni, and A. Jaddar, On generalized monotone multifunctions with applications to optimality conditions in generalized convex programming, JIPAM, J. Inequal. Pure Appl. Math. 4, No.4, Paper No.67, 11 p.

[127] A. Hassouni and A. Jaddar, Quasi-convex functions and applications to optimality conditions in nonlinear programming, Appl. Math. Lett. 14, No.2, 241-244 (2001).

[128] A. Hassouni and A. Jaddar, On pseudoconvex functions and applications to global optimization, preprint, Univ. Mohamed V, Rabat (2004).

[129] R. Horst and P.M. Pardalos, Handbook of global optimization, Nonconvex Optim. and its Appli. 2, Kluwer, Dordrecht (1995).

[130] R. Horst and N.V. Thoai, DC programming: Overview, J. Optim. Theory Appl. 103, No.1 (1999), 1-43.

[131] R. Horst and H. Tuy, Global optimization. Deterministic approaches. 3rd ed, Springer, Berlin (1996).

[132] A.D. Ioffe, On subdifferentiability spaces, Ann. N.Y. Acad. Sci. 410 (1983), 107-119

[133] A.D. Ioffe, Subdifferentiability spaces and nonsmooth analysis, Bull. Amer. Math. Soc. 10 (1984), 87-90.

[134] A.D. Ioffe, Approximate subdifferentials and applications. III: The metric theory, Mathematika 36, No.1 (1989), 1-38.

[135] A.D. Ioffe, Proximal analysis and approximate subdifferentials, J. London Math. Soc. 41 (1990), $261-268$.

[136] A.D. Ioffe, Abstract convexity and non-smooth analysis, Advances Math. Econ. 3, 45-61 (2001).

[137] V.I. Ivanov, First order characterizations of pseudoconvex functions, Serdica Math. J. 27 (2001), $203-218$.

[138] A. Jofré, D.T. Luc and M. Théra, Subdifferential and monotonicity, Nonlinear Anal. 33 (1998), no. 1, 71-90.

[139] R. John, A first order characterization of generalized monotonicity, Math. Program. A 88 (2000), $147-155$.

[140] R. John, Uses of generalized convexity and generalized monotonicity in economics, Handbook on Generalized Convexity and Monotonicity, N. Hadjisavvas and S. Schaible (eds.), 619-666, Kluwer, Dordrecht (2004).

[141] C. Jouron, On some structural design problems, Bull. Soc. Math. Fr., Suppl., Mém. 60 (Proc. Colloq., Pau 1977), 87-93 (1979).

[142] M. Jovanovic', Some inequalities for strong quasiconvex functions, Glasnik Mat. 24 (44) (1989), $25-29$.

[143] Z. A. Khan, On nondifferentiable quasiconvex programming problem, J. Inf. Optimization Sci. 12, (1) (1991), 57-64.

[144] S. Karamardian, Complementarity over cones with monotone and pseudomonotone maps, J. Optim. Theory Appl. 18 (1976), 445-454.

[145] S. Karamardian and S. Schaible, Seven kinds of monotone maps, J. Optim. Theory Appl. 66 (1990), $37-46$.

[146] S. Karamardian, S. Schaible and J.-P. Crouzeix, Characterizations of generalized monotone maps, J. Opt. Th. Appl. 76 (3) (1993), 399-413. 
[147] V.G. Karmanov, Programmation mathématique, Mir, Moscow, 1977.

[148] G. Kassay, J. Kolumbán, On a generalized sup-inf problem, J. Optim. Theory Appl. 91 (3), (1996), 651-670.

[149] K.C. Kiwiel, Convergence and efficiency of subgradient methods for quasiconvex minimization, Math. Program. 90, No.1(A) (2001), 1-25.

[150] S. Komlosi, Some properties of nondifferentiable pseudoconvex functions, Math. Programming 26 (1983), 232-237.

[151] S. Komlosi, On generalized upper quasidifferentiability, in Nonsmooth Optimization : Methods and Applications, F. Giannessi (ed.), Gordon and Breach, London, 1992.

[152] S. Komlósi, On pseudoconvex functions, Acta Sci. Math. 57, No.1-4, 569-586 (1993).

[153] S. Komlósi, Quasiconvex first-order approximations and Kuhn-Tucker type optimality conditions, Eur. J. Oper. Res. 65, No.3, 327-335 (1993).

[154] S. Komlósi, First and second order characterizations of pseudolinear functions, European J. Oper. Research 67 (1993), $278-293$.

[155] S. Komlósi, T. Rapcsák and S. Schaible, Generalized Convexity. Proceedings of the 5th international workshop on generalized convexity, Pécs, Hungary, 1992, Lecture Notes in Econ. and Math. Systems \# 405 Springer-Verlag, Berlin (1994).

[156] S. Komlósi, Monotonicity and quasimonotonicity in nonsmooth analysis, Recent advances in nonsmooth optimization, Du, Ding-Zhu et al., (eds.) World Scientific, Singapore, 193-214 (1995).

[157] S. Komlosi, Generalized monotonicity and generalized convexity, J. Optim. Theory Appl. 84, No.2, 361-376 (1995).

[158] K.O. Kortanek and J.P. Evans, Pseudo-concave programming and Lagrange regularity, Operations Research 15, (1967), 882-892.

[159] S. Kruk and H. Wolkowicz, Pseudoconvex functions, SIAM Review 41 (4) (1999), 795-805.

[160] B. Lemaire and M. Volle, Duality in DC programming, in Generalized Convexity, Generalized Monotonicity, Recent Results, J.-P. Crouzeix et al (Eds.) Kluwer, Dordrecht (1988), 331-345.

[161] Levin, V., Abstract cyclical monotonicity and Monge solutions for the general Monge-Kantorovich problem, Set-Valued Anal. 7, No.1, 7-32 (1999).

[162] Levin, V.L., Semiconic duality in convex analysis, Trans. Mosc. Math. Soc. 2000, 197-238 (2000); translation from Tr. Mosk. Mat. O.-va 61, 210-253 (2000).

[163] Levin, V.L., Abstract convexity in measure theory and in convex analysis, J. Math. Sci., New York 116, No.4, 3432-3467 (2003).

[164] N.T.H. Linh and J.-P. Penot, Optimality conditions for quasiconvex programs, SIAM J. Optim. 17 (2) (2006), 500-510.

[165] N.T.H. Linh and J.-P. Penot, Generalized affine maps and generalized convex functions, preprint, Univ. of Pau, May 2006.

[166] N.T.H. Linh and J.-P. Penot, Generalized convex functions and generalized differentials, preprint, Univ. of Pau, July 2006.

[167] N.T.H. Linh and J.-P. Penot, Generalized affine maps and generalized differentials, preprint, Univ. of Pau, October 2006.

[168] N.T.H. Linh and J.-P. Penot, Approximately quasiconvex functions, in preparation.

[169] Ph. D. Loewen, A mean value theorem for Fréchet subgradients, Nonlinear Anal., Theory Methods Appl. 23 , No.11 (1994), 1365-1381.

[170] D.T. Luc, Characterizations of quasiconvex functions, Bull. Austral. Math. Soc. 48 (1993), 393-405.

[171] D.T. Luc, On generalized convex nonsmooth functions, Bull. Austral. Math. Soc. 49 (1993), 139-149.

[172] D.T. Luc and J.-P. Penot, Convergence of asymptotic directions, Trans. Amer. Math. Soc. 353 (2001), 4095-4121.

[173] D.T. Luc and S. Schaible, Generalized monotone nonsmooth maps, J. Convex Anal. 3 (2) (1996), 195-206.

[174] D.T. Luc and S. Swaminathan, A characterization of convex functions, Nonlinear Analysis, Theory, Methods \& Appl., 20 (1993), 697-701.

[175] D.T. Luc and M. Volle, Level set under infimal convolution and level addition, J. Opt. Th. Appl. 94, No.3, 695-714 (1997).

[176] V. Lyall, S. Suneja, S. Aggarval, Optimality and duality in fractional programming involving semilocally convex and related functions, Optim. 41 (1997), 237-255.

[177] O.L. Mangasarian, Pseudoconvex functions, SIAM J. Control 3 (1965), 281-290.

[178] O.L. Mangasarian, Nonlinear Programming, Mc Graw-Hill, New-York, 1969.

[179] D.H. Martin, The essence of invexity, J. Optimization Theory Appl. 47, 65-76 (1985).

[180] J.-E. Martínez-Legaz, A generalized concept of conjugation methods, in Optimization, theory and algorithms, J.-B. HiriartUrruty, W. Oettli, J. Stoer, (eds.), Dekker, New York (1983), 45-49.

[181] J.-E. Martínez-Legaz, On lower subdifferentiable functions, in Trends in Mathematical Optimization, K.H. Hoffmann et al. (eds), Int. Series Numer. Math. 84, Birkhauser, Basel (1988) 197-232.

[182] J.-E. Martínez-Legaz, Quasiconvex duality theory by generalized conjugation methods, Optimization, 19 (1988) 603-652.

[183] J.-E. Martínez-Legaz, Weak lower subdifferentials and applications, Optimization 21 (1990) 321-341.

[184] J.-E. Martínez-Legaz, Fenchel duality and related properties in generalized conjugaison theory, S.E.A. Bulletin Math. 19 (2) (1995), 99-106.

[185] J.-E. Martínez-Legaz, On convex and quasiconvex spectral functions, in "Proceedings of the second Catalan days on Applied Mathematics", M. Sofonea and J.-N. Corvellec, eds., Presses Univ. Perpignan (1995), 199-208.

[186] J.-E. Martínez-Legaz, Generalized convex duality and its economic applications, Handbook on Generalized Convexity and Monotonicity, N. Hadjisavvas and S. Schaible (eds.), 237-292, Kluwer, Dordrecht (2004).

[187] J.-E. Martínez-Legaz and S. Romano-Rodríguez, $\alpha$-lower subdifferentiable functions, SIAM J. Optim. 3 (1993) $800-825$. 
[188] J.-E. Martínez-Legaz and S. Romano-Rodríguez, Lower subdifferentiability of quadratic functions, Math. Programming 60 (1993) 93-113.

[189] J.-E. Martínez-Legaz and I. Singer, Subdifferentials with respect to dualities, ZOR-Math. Methods Oper. Res. 42 (1995), $109-125$.

[190] B. Martos, Nonlinear Programming. Theory and methods, North-Holland, Amsterdam (1975).

[191] S.K. Mishra, Pseudolinear fractional minimax programming, Indian J. Math. Pure Appl. Math. 26 (8), (1995), $722-763$.

[192] B. S. Mordukhovich, Variational analysis and generalized differentiation. I. Basic theory. Grundlehren der Mathematischen Wissenschaften, 330. Springer-Verlag, Berlin, 2006.

[193] B. S. Mordukhovich, Variational analysis and generalized differentiation. II. Applications. Grundlehren der Mathematischen Wissenschaften, 331. Springer-Verlag, Berlin, 2006.

[194] J.-J. Moreau, Inf-convolution, sous-additivité, convexité des fonctions numériques, J. Math. Pures Appl. 49 , 109-154 (1970).

[195] K. Murota, Discrete Convex Analysis, SIAM Monographs on Discrete Maths. and Appl. SIAM, Philadelphia (2003).

[196] Yu. E. Nesterov, Minimization methods for nonsmooth convex and quasiconvex functions, Ekkonom. i Mat. Metody 20 (1984), 519-531 (in Russian). English transl. in Matekon 29 (3) (1984), 519-531.

[197] H.V. Ngai, D.T. Luc and M. Théra, Approximate convex functions, J. Nonlinear and Convex Anal. 1 (2) (2000), 155-176.

[198] H.V. Ngai and J.-P. Penot, Semismoothness and directional subconvexity of functions, preprint, May 2003, revised May 2005, to appear in Pacific J. Optimization.

[199] H.V. Ngai and J.-P. Penot, Approximately convex functions and approximately monotone operators, Nonlinear Analysis 66 (2007), 547-564.

[200] H.V. Ngai and J.-P. Penot, Rambling through generalized convexity and generalized monotonicity, in Generalized Convexity and Generalized Monotonicity, I.V. Konnov, D.T. Luc, A.M. Rubinov (eds.) Lecture Notes in Economics and Mathematical Systems 583, Springer, Berlin (2007), 379-397.

[201] H.V. Ngai and J.-P. Penot, Approximately convex sets, preprint, October 2004.

[202] H.V. Ngai and J.-P. Penot, Paraconvex functions and paraconvex sets, preprint, October 2004.

[203] H.V. Ngai and J.-P. Penot, In Asplund spaces, approximately convex functions are generically differentiable, preprint, November 2005

[204] W. Oettli, D. Schläger, Conjugate functions for convex and nonconvex duality, J. Global Optim. 13 (1998), $337-347$.

[205] D. Pallaschke and S. Rolewicz, Foundations of mathematical optimization. Convex analysis without linearity. Maths. and its appl. , vol. 388, Kluwer, Dordrecht (1998).

[206] P. D. Panagiotopoulos, Nonsmooth/nonconvex mechanics (Blacksburg, VA, 1999), Nonconvex Optim. Appl., 50, Kluwer, Dordrecht, 2001.

[207] U. Passy and E.Z. Prisman, A convexlike duality scheme for quasiconvex programs, Math. Programming 32 (1985), 278-300.

[208] J.-P. Penot, Mean-value theorem with small subdifferentials, J. Opt. Th. Appl. 94 (1) (1997), $209-221$.

[209] J.-P. Penot, Favorable classes of mappings and multimappings in nonlinear analysis and optimization, J. Convex Analysis 3 (1) (1996), 97-116.

[210] J.-P. Penot, Well-behavior, well-posedness and nonsmooth analysis, Pliska Stud. Math. Bulgar. 12 (1998), $141-190$.

[211] J.-P. Penot, Are generalized derivatives useful for generalized convex functions?, in Generalized convexity, generalized monotonicity: recent results, J.-P. Crouzeix, J.E. Martínez-Legaz and M. Volle (eds), Kluwer, Dordrecht (1998) 3-59.

[212] J.-P. Penot, On the minimization of difference functions, J. Global Optim. 12 (1998), 373-382.

[213] J.-P. Penot, What is quasiconvex analysis?, Optimization 47 (2000), 35-110.

[214] J.-P. Penot, A variational subdifferential for quasiconvex functions, J. Optim. Th. Appl. 111 (1), (2001), $165-171$.

[215] J.-P. Penot, Characterization of solution sets of quasiconvex programs, J. Optim. Th. Appl. 117 (3) (2003) 627-636.

[216] J.-P. Penot, A Lagrangian approach to quasiconvex analysis, J. Optim. Th. Appl. 117 (3) (2003), $637-647$.

[217] J.-P. Penot, The bearing of duality on microeconomics, Advances in Math. Econ. 7 (2005), 113-139.

[218] J.-P. Penot, Unilateral analysis and duality, in Essays and Surveys in Global Optimization for the 25th anniversary of GERAD, G. Savard et al eds, Springer, New York (2005), 1-37.

[219] J.-P. Penot, Noncoercive problems and asymptotic conditions, Asymptot. Anal. 49 no. 3-4, (2006), 205-215.

[220] J.-P. Penot, Softness, sleekness and regularity properties in nonsmooth analysis, to appear in Nonlinear Analysis.

[221] J.-P. Penot, Monotonicity and duality, in Generalized Convexity and Generalized Monotonicity, I.V. Konnov, D.T. Luc, A.M. Rubinov (eds.) Lecture Notes in Economics and Mathematical Systems 583, Springer, Berlin (2007), 399-414.

[222] J.-P. Penot, Gap continuity of multimaps, preprint, Sept. 2005.

[223] J.-P. Penot, Critical duality, preprint, Univ. of Pau, 2006.

[224] J.-P. Penot and M. Bougeard, Approximation and decomposition properties of some classes of locally d.c. functions, Math. Programming 41 (1988), 195-227.

[225] J.-P. Penot and P.H. Quang, On generalized convex functions and generalized monotonicity of set-valued maps, J. Optim. Theory Appl. 92, No.2, 343-356 (1997).

[226] J.-P. Penot and P.H. Quang, Cutting planes algorithms and approximate lower subdifferentiability, to appear in J. Optim. Th. Appl.

[227] J.-P. Penot and A.M. Rubinov, Multipliers and general Lagrangians, Optimization 54, No.4-5 (2005), 443-467. 
[228] J.-P. Penot and P. H. Sach, Generalized monotonicity of subdifferentials and generalized convexity, J. Optim. Th. and Appl. 94 (1) (1997), 251-262.

[229] J.-P. Penot and M. Volle, Dualité de Fenchel et quasi-convexité, C.R. Acad. Sci. Paris Série I 304 (13) (1987), 371-374.

[230] J.-P. Penot and M. Volle, Another duality scheme for quasiconvex problems, Trends in Mathematical Optimization, K.H. Hoffmann et al. eds, Int. Series Numer. Math. 84 Birkhauser, Basel, (1988), 259-275.

[231] J.-P. Penot and M. Volle, On quasi-convex duality, Math. Oper. Research 15 (4) (1990), 597-625.

[232] J.-P. Penot and M. Volle, Surrogate programming and multipliers in quasiconvex programming, SIAM J. Control Optim. 42 (6)(2003), 1994-2003.

[233] J.-P. Penot and M. Volle, Explicit solutions to Hamilton-Jacobi equations under mild continuity and convexity assumptions, J. Nonlinear Convex Anal. 1, No.2, 177-199 (2000).

[234] J.-P. Penot and M. Volle, Convexity and generalized convexity methods for the study of Hamilton-Jacobi equations, in Generalized convexity and generalized monotonicity. Proceedings of the 6th international symposium, Samos, Greece, 1999, N. Hadjisavvas et al., (eds.), Lect. Notes Econ. Math. Syst. 502, Springer, Berlin, 294-316 (2001).

[235] J.-P. Penot and M. Volle, Duality methods for the study of Hamilton-Jacobi equations, ESAIM Proc., to appear.

[236] J.-P. Penot and C. Zălinescu, Elements of quasiconvex subdifferential calculus, J. of Convex Analysis 7 (2000), $243-269$.

[237] J.-P. Penot and C. Zălinescu, Harmonic sums and duality, J. of Convex Analysis 7 (2000) 95-113.

[238] J.-P. Penot and C. Zălinescu, Elements of quasiconvex subdifferential calculus, J. of Convex Analysis 7 (2000) 243-269.

[239] J.-P. Penot and C. Zălinescu, Approximation of functions and sets, in Approximation, optimization and mathematical economics. Proceedings of the 5th international conference on "Approximation and optimization in the Caribbean", M. Lassonde, (ed.), Physica-Verlag, Heidelberg, 255-274 (2001).

[240] J.-P. Penot and C. Zălinescu, Continuity of usual operations and variational convergences, Set-Valued Analysis 11 (3) (2003), 225-256.

[241] J.-P. Penot and C. Zălinescu, Persistence and stability of explicit solutions of Hamilton-Jacobi equations, submitted.

[242] R.R. Phelps, Convex functions, monotone operators and differentiability. 2nd ed., Lecture Notes in Maths. 1364, SpringerVerlag, Berlin (1993).

[243] Phu, H.X., An, P.T., Stable generalization of convex functions, Optimization 38 (4) (1996), 309-318.

[244] R. Pini, Invexity and generalized convexity. Optimization 22 (1991), no. 4, 513-525.

[245] R. Pini, Convexity along curves and invexity. Optimization 29 (1994), no. 4, 301-309.

[246] R. Pini, A note on P-convexity. J. Global Optim. 5 (1) (1994), 15-20.

[247] R. Pini and S. Schaible, Invariance properties of generalized monotonicity. Optimization 28 (1994), no. 3-4, $211-222$.

[248] R. Pini, C. Singh, Sectionally convexible functions. J. Inform. Optim. Sci. 16 (1995), no. 3, 461-470.

[249] R. Pini, C. Singh, Generalized convexity and generalized monotonocity. J. Inform. Optim. Sci. 20 (1999), no. 2, 215-233.

[250] R. Pini, C. Singh, A survey of recent [1985-1995] advances in generalized convexity with applications to duality theory and optimality conditions, Optimization 39 (4) (1997), 311-360.

[251] F. Plastria, Lower subdifferentiable functions and their minimization by cutting plane, J. Opt. Th. Appl. 46 (1) (1985) 37-54.

[252] R.A. Poliquin, Subgradient monotonicity and convex functions, Nonlin. Anal., Theory, Meth. and Appl. 14 (1990), 305-317.

[253] J. Ponstein, Seven kinds of convexity, SIAM Rev. 9 (1967), 115-119.

[254] T. Rapcsák, On pseudolinear functions, European J. of Oper. Research 50 (1991), 353-360.

[255] R.T. Rockafellar, Favorable classes of Lipschitz continuous functions in subgradient optimization, in Nondifferentiable Optimization (1982), Nurminski E. (eds), Pergamon Press, New York.

[256] R.T. Rockafellar and R. J-B. Wets, Variational Analysis, Springer-Verlag, Berlin, 1997.

[257] S. Rolewicz, Convex analysis without linearity, Control and Cybern. 23 (1994), 247-256.

[258] S. Rolewicz, Duality and convex analysis in the absence of linear structure, Math. Japonica 44 (1996), 165-182.

[259] A.M. Rubinov, Abstract Convexity and Global Optimization, Kluwer, Dordrecht (2000).

[260] A.M. Rubinov, Radiant sets and their gauges, in Quasidifferentiability and Related Topics, V. Demyanov and A.M. Rubinov, eds. Kluwer, Dordrecht (2000).

[261] A.M. Rubinov and M. Andramonov, Lipschitz programming via increasing convex-along-rays functions, Optim. Methods Softw. 10, pp. 763-781 (1999).

[262] A.M. Rubinov and B.M. Glover, On generalized quasiconvex conjugation, in Recent Developments in Optimization Theory and Nonlinear Analysis, Y. Censor and S. Reich, eds., American Mathematical Society, Providence, pp. 199-216 (1997).

[263] A.M. Rubinov and B.M. Glover, Quasiconvexity via two steps functions, in Generalized Convexity, Generalized Monotonicity, J.-P. Crouzeix, J.-E. Martínez-Legaz and M. Volle, eds., Kluwer Academic Publishers, Dordrecht (1998), 159-183.

[264] A.M. Rubinov and B.M. Glover, Duality for increasing positively homogeneous functions and normal sets, Rech. Opér 32 (1998), 105-123.

[265] A.M. Rubinov and B.M. Glover, Increasing convex-along-rays functions with applications to global optimization, J. Optim. Theory Appl. 102, pp. 615-642 (1999).

[266] A.M. Rubinov, B.M. Glover and X.Q. Yang, Extended Lagrange and penalty functions in continuous optimization, Optimization, 46 (1999), 327-351. 
[267] A.M. Rubinov, B.M. Glover and X.Q. Yang, Decreasing functions with applications to optimization, SIAM J. Optim. 10 (1999), 289-313.

[268] A.M. Rubinov, B.M. Glover and X.Q. Yang, Nonlinear unconstrained optimization methods: a review, in X. Yang et al. (eds.), Progress in Optimization, Kluwer, Dordrecht, (2000), 65-77.

[269] A.M. Rubinov and B. Simsek, Conjugate quasiconvex nonnegative functions, Optimization, 35, 1-22 (1995).

[270] A.M. Rubinov and B. Simsek, Dual problems of quasiconvex maximization, Bull. Aust. Math. Soc. 51, 139-144 (1995).

[271] A.M. Rubinov and B. Simsek, Separability of star-shaped sets with respect to infinity, Progress in Optimization, X. Yang et al. (eds.), Kluwer, Dordrecht (2000), 45-63.

[272] N.G. Rueda, M.A. Hanson and C. Singh, Optimality and duality with generalized convexity, J. Optim. Theory Appl. 86, No.2, 491-500 (1995).

[273] P.H. Sach and J.-P. Penot, Characterizations of generalized convexities via generalized directional derivatives, Numer. Funct. Anal. Optimization 19, No.5-6, 615-634 (1998).

[274] J.A. dos Santos Gromicho, Quasiconvex optimization and location theory, Kluwer, Dordrecht (1998).

[275] S. Schaible, Fractional programming, I, Duality, Management Sci. 22 (1976) 858-867.

[276] S. Schaible, Duality in fractional programming: a unified approach, Operations Research 24 (3) (1976) 452-461.

[277] S. Schaible, Second-order characterizations of pseudoconvex quadratic functions, J. Opt. Th. Appl. 21 (1) (1977), 15-26.

[278] S. Schaible, Generalized monotone maps, in Nonsmooth optimization: Methods and Applications, Proc. Symp. Erice, June 1991, F. Giannessi (ed.) Gordon and Breach, Amsterdam, 1992, 392-408.

[279] S. Schaible, Generalized monotonicity-a survey, in Generalized convexity, Proc. Pecs, Hungary 1992, Lecture Notes in Economics and Math. Systems \#405, S. Komlósi, T. Rapscák, S. Schaible (eds.), Springer-Verlag, Berlin (1994), 229-249.

[280] S. Schaible, Generalized monotonicity-concepts and uses, in Variational inequalities and network equilibrium problems, Proc. 19th course, Int. School of Math. Erice, June 1994, F. Giannessi and A. Maugeri, eds. Plenum, New York, 1995, $289-299$.

[281] S. Schaible and W.T. Ziemba (eds.), Generalized Concavity in Optimization and Economics, Academic Press, New-York, 1981.

[282] E.A. Sideri, A new local characterization of pseudoconvex functions and their nonsmooth extensions, in Nonsmooth optimization: methods and applications. Proceedings of the meeting "G. Stampacchia International School of Mathematics", Erice, Italy, Giannessi, F. (ed.) Gordon and Breach Amsterdam (1992), 409-420.

[283] S. Simons, Minimax theorems and their proofs, in "Minimax and applications", Ding-Zhu Du and Panos M. Pardalos eds., Kluwer Academic Publishers, Dordrecht, Boston (1995), 1-23.

[284] S. Simons, Minimax and monotonicity, Lecture Notes in Maths \#1693 Springer, Berlin, (1998).

[285] I. Singer, Some relations between dualities, polarities, coupling functions and conjugations, J. Math. Anal. Appl. 115 (1986), $1-22$.

[286] I. Singer, Abstract Convex Analysis, Wiley, New York (1997).

[287] M. Sion, On general minimax theorems, Pacific J. Math. 8 (1958), 171-176.

[288] M.J. Śmietański, Kuhn-Tucker type optimality conditions for some class of nonsmooth programming problems, Control \& Cybernetics 32 (2) (2003), 361-376.

[289] J.E. Spingarn, Submonotone subdifferentials of Lipschitz functions, Trans. Am. Math. Soc. 264, 77-89 (1981).

[290] C. Sutti, Quasidifferentiability of nonsmooth quasiconvex functions, Optimization 27 (4) (1993) 313-320.

[291] Y. Tanaka, Note on generalized convex functions, J. Optim. Th. Appl. 66 (2) (1990) 345-349.

[292] P.D. Tao and S. El Bernoussi, Duality in D.C. (difference of convex functions). Optimization. Subgradient methods, in Trends in Mathematical Optimization, K.H. Hoffmann et al. eds, Int. Series Numer. Math. \# 84, Birkhäuser, Basel, 1988, $277-293$.

[293] P.D. Tao and Le Thi Hoai An, Convex analysis approach to D.C. programming: theory, algorithms and applications, Acta Math. Vietnamica 22 (1) (1997), 289-355.

[294] P.T. Thach, Quasiconjugate of functions, duality relationships between quasiconvex minimization under a reverse convex convex constraint and quasiconvex maximization under a convex constraint and application, J. Math. Anal. Appl. 159 (1991) 299-322.

[295] P.T. Thach, Global optimality criterion and a duality with a zero gap in nonconvex optimization, SIAM J. Math. Anal. 24 (6) (1993), 1537-1556.

[296] P.T. Thach, A nonconvex duality with zero gap and applications, SIAM J. Optim. 4 (1) (1994), 44-64.

[297] P.T. Thach and M. Kojima, A generalized convexity and variational inequality for quasi-convex minimization, SIAM J. Optim. 6 (1) (1996), 212-226.

[298] L. Thibault and D. Zagrodny, Integration of subdifferentials of lower semi-continuous functions on Banach spaces, J. Math. Anal. and Appl. 189 (1995), 33-58.

[299] W.A. Thompson, Jr. and D.W. Parke, Some properties of generalized concave functions, Oper. Research 21 (1) (1974), 305-313.

[300] S. Traoré and M. Volle, Quasi-convex conjugation and Mosco convergence, Richerche di Mat. 44 (2) (1995), 369-388.

[301] S. Traoré and M. Volle, Subdifferentiability, lower semicontinuity and exactness of the level sum of two convex functions on locally convex spaces, in Recent Developments in Optimization, R. Durier and C. Michelot eds., Lecture Notes in Economics and Mathematical Systems 429, Springer Verlag, Berlin, (1995), 269-290. 
[302] S. Traoré and M. Volle, On the level sum of two convex functions on Banach spaces, J. Convex Anal. 3 (1) (1996), $141-151$.

[303] S. Traoré and M. Volle, Epiconvergence d'une suite de sommes en niveaux de fonctions convexes, Serdica Math. J. 22 (1996), 293-306.

[304] H. Tuy, Convex programs with an additional reverse convex constraint, J. Optim. Theory Appl. 52 (1987), $463-486$.

[305] H. Tuy, D.C. optimization: theory, methods and algorithms, in Handbook of Global Optimization, R. Horst and P.M. Pardalos, eds., Kluwer, Dordrecht, Netherlands (1995), 149-216.

[306] H. Tuy, On nonconvex optimisation problems with separated nonconvex variables, J. Global Optim. 2 (1992), $133-144$.

[307] H. Tuy, Convex Analysis and Global Optimization, Kluwer, Dordrecht, (1998).

[308] J.-P. Vial, Strong and weak convexity of sets and functions, Math. Oper. Res. 8 (2) (1983) $231-259$.

[309] A.A. Vladimirov, Yu.E. Nesterov and Yu.N. Chekanov, On uniformly convex functionals, Mosc. Univ. Comput. Math. Cybern. 1978, No.3, 10-21 (1978); translation from Vestn. Mosk. Univ., Ser. XV 1978, No.3, 12-23 (1978).

[310] A.A. Vladimirov, Yu.E. Nesterov and Yu.N. Chekanov, Uniformly quasi convex functionals, Vestn. Mosk. Univ., 1978, No.4, 18-27 (1978).

[311] A.A. Vladimirov, Yu.E. Nesterov and Yu.N. Chekanov, On uniformly quasi-convex functionals, Mosc. Univ. Comput. Math. Cybern. 1978, No.4, 19-30 (1978); translation from Vestn. Mosk. Univ., Ser. XV 1978, No.4, 18-27 (1978).

[312] M. Volle, Convergence en niveaux et en épigraphes, C.R. Acad. Sci. Paris 299 (8) (1984), pp. $295-298$.

[313] M. Volle, Conjugaison par tranches, Annali Mat. Pura Appl. 139 (1985) 279-312.

[314] M. Volle, Contributions à la dualité en optimisation et à l'épiconvergence, Thesis, Univ. Pau, (1986).

[315] M. Volle, Conjugaison par tranches et dualité de Toland, Optimization 18 (1987) 633-642.

[316] M. Volle, Quasiconvex duality for the max of two functions, in Recent advances in optimization, P. Gritzmann, R. Horst, E. Sachs, R. Tichatschke (eds.) Lecture Notes in Economics and Math. Systems 452 (1997), 365-379.

[317] M. Volle, Conditions initiales quasiconvexes dans les équations de Hamilton-Jacobi, C.R. Acad. Sci. Paris série I, 325 (1997), $167-170$

[318] M. Volle, Duality for the level sum of quasiconvex functions and applications, ESAIM: Control, Optimisation and Calculus of Variations, http://www.emath.fr/cocv/ 3 (1998), 329-343.

[319] R. J-B. Wets, A formula for the level sets of epi-limits and some applications, in Theories of Optimization. Proceedings 1981, J.P. Cecconi and T. Zolezzi (eds.), Lecture Notes in Maths \# 979 (1983), 256-268.

[320] X. M. Yang, Semistrictly convex functions, Opsearch 31 (1994), 15-27.

[321] X.Q. Yang, Generalized convex functions and vector variational inequalities, J. Opt. Th. and Appl., 79 (1993) 563-580.

[322] X.Q. Yang, Generalized second-order characterizations of convex functions, J. Opt. Th. and Appl., 82 (1994) $173-180$.

[323] X.Q. Yang, Continuous generalized convex functions and their characterizations, Optimization 54 (4-5) (2005), 495-506.

[324] X.Q. Yang and Guang-Ya Chen, A class of nonconvex functions and pre-variational inequalities, J. Math. Anal. Appl. 169, No.2, 359-373 (1992).

[325] J.C. Yao, Multivalued variational inequalities with K-pseudomonotone operators, J. Opt. Th. and Appl. 83 (1994), $391-401$.

[326] A. Zaffaroni, Is every radiant function the sum of quasiconvex functions? Math. Methods Oper. Res. 59 (2004), $221-233$.

[327] D. Zagrodny, General sufficient conditions for the convexity of a function, Zeitschrift fur Anal. Anwendungen 11 (1992), $277-283$.

[328] D. Zagrodny, Some recent mean value theorems in nonsmooth analysis, in Nonsmooth Optimization. Methods and Applications, Proc. Symp. Erice 1991, F. Giannessi ed., Gordon and Breach, OPA, Amsterdam 1992, $421-428$.

[329] D. Zagrodny, General sufficient conditions for the convexity of a function, Zeitschrift fur Anal. Anwendungen 11 (1992), $277-283$.

[330] I. Zang and M. Avriel, On functions whose local minima are global, J. Optim. Theory Appl. 16, 183-190 (1975).

[331] I. Zang, E.U. Choo and M. Avriel, A note on functions whose local minima are global, J. Optim. Theory Appl. 18, 555-559 (1976). 